\title{
Studies on the Syntheses of Benzoquinone
}

\section{Ansamycin Antibiotics. Syntheses of the}

\section{C(5)-C(15) Subunits of Macbecin I, \\ Geldanamycin and Herbimycin A}

\author{
Justin K. Belardi and Glenn C. Micalizio* \\ Department of Chemistry, Yale University, New Haven, CT 06520-8107 \\ E-mail: glenn.micalizio@yale.edu
}

SUPPORTING INFORMATION: 
General. All reactions were conducted in flame-dried glassware under nitrogen using anhydrous solvents. Toluene was dried by distillation over $\mathrm{CaH}_{2}$. Diisopropylamine was dried by distillation over $\mathrm{NaOH}$. Methylene chloride, tetrahydrofuran and diethyl ether were used after passing through activated alumina columns. All other commercially available reagents were used as received.

${ }^{1} \mathrm{H}$ NMR data were recorded at $500 \mathrm{MHz}$ or $400 \mathrm{MHz}$ using a Bruker AM-500, Bruker Avance DPX-500 or Bruker AM-400 instrument. ${ }^{1} \mathrm{H}$ NMR chemical shifts are reported relative to residual $\mathrm{CHCl}_{3}(7.26 \mathrm{ppm}) .{ }^{13} \mathrm{C}$ NMR data were recorded at $126 \mathrm{MHz}$ or $100 \mathrm{MHz}$ using a Bruker AM-500, Bruker Avance DPX-500 or Bruker AM-400 instrument. ${ }^{13} \mathrm{C}$ chemical shifts are reported relative to the central line of $\mathrm{CDCl}_{3}(77.23 \mathrm{ppm})$. Infrared spectra were recorded using a Thermo Electron Nicolet 6700 FT-IR Spectrometer. Low resolution mass spectrometry was performed on a Waters Micromass ${ }^{\circledR} \mathrm{ZQ}^{\mathrm{TM}}$ instrument using electrospray ionization (EI) or chemical ionization (CI). Optical rotations were measured on Perkin Elmer Model 341 polarimeter using a $1 \mathrm{~mL}$ capacity micro cell with a $10 \mathrm{~cm}$ path length. Chromatographic purifications were performed using $60 \AA, 35-75 \mu \mathrm{m}$ particle size silica gel from Silicycle. All compounds purified by chromatography were sufficiently pure for use in further experiments, unless indicated otherwise. Semi-preparative and analytical HPLC normal phase separations were performed using an HPLC system composed of two Dynamax SD-1 pumps, a Rheodyne injector and a Dynamax UV-1 absorbance detector. 


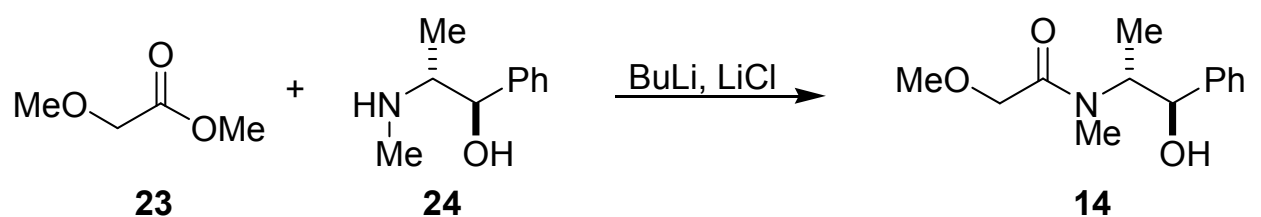

Synthesis of $\quad(R, R)-\alpha$-methoxy- $N$-(2-hydroxy-1-methyl-2-phenylethyl)- $N$ -

methylacetamide, 14: A solution of $n$-butyllithium in hexanes $(2.5 \mathrm{M}, 12.1 \mathrm{~mL}, 30.3 \mathrm{mmol})$ was added to an ice-cooled suspension of lithium chloride (7.7 $\mathrm{g}, 181 \mathrm{mmol})$ and (-)pseudoephedrine $24(10 \mathrm{~g}, 60.5 \mathrm{mmol})$ in tetrahydrofuran $(400 \mathrm{~mL})$, and the suspension was stirred at $0{ }^{\circ} \mathrm{C}$ for $30 \mathrm{~min}$. Methyl methoxyacetate $23(12.0 \mathrm{~mL}, 121 \mathrm{mmol})$ was added via syringe over $5 \mathrm{~min}$, and the mixture was warmed to $23{ }^{\circ} \mathrm{C}$ and stirred at that temperature for 3 h. A solution of $0.5 \mathrm{~N}$ aqueous sodium hydroxide $(200 \mathrm{~mL})$ was added, and the biphasic mixture was stirred at $23{ }^{\circ} \mathrm{C}$ for $1 \mathrm{~h}$. Volatile organic solvents were removed under reduced pressure, and the resulting aqueous residue was extracted with five $50-\mathrm{mL}$ portions of $10 \%$ methanol-dichloromethane. The combined organic extracts were dried over sodium sulfate and concentrated. Purification of the product by flash column chromatography eluting with methanol-dichloromethane (8\%) afforded amide 14 as a colorless oil which slowly solidified. The solid was subsequently recrystallized from hot toluene furnishing pure crystalline amide 14 as a 1.3:1 mixture of rotamers $(12.6 \mathrm{~g}, 53.1 \mathrm{mmol}, 88 \%)$.

Data for $(R, R)-\alpha$-methoxy- $N$-(2-hydroxy-1-methyl-2-phenylethyl)- $N$-methylacetamide, 14: $[\alpha]_{589}{ }^{20}-98.7^{\circ}\left(c=1.51, \mathrm{CHCl}_{3}\right) ;{ }^{1} \mathrm{H} \mathrm{NMR}\left(500 \mathrm{MHz}, \mathrm{CDCl}_{3}\right) \delta 7.39-7.24(\mathrm{~m}, 10 \mathrm{H})$, 4.62 (app. t, $J=7.6 \mathrm{~Hz}, 1 \mathrm{H}), 4.51$ (dd, $J=9.1,4.7 \mathrm{~Hz}, 1 \mathrm{H}), 4.46-4.38(\mathrm{~m}, 1 \mathrm{H}), 4.22-4.16$ (m, 2H), 4.10-3.96 (m, 4H), 3.45 (s, 3H), $3.37(\mathrm{~s}, 3 \mathrm{H}), 3.19$ (d, J=4.4 Hz, 1H), $2.93(\mathrm{~s}, 3 \mathrm{H}), 2.80$ $(\mathrm{s}, 3 \mathrm{H}), 1.12(\mathrm{~d}, J=6.9 \mathrm{~Hz}, 3 \mathrm{H}), 0.98(\mathrm{~d} J=6.6 \mathrm{~Hz}, 3 \mathrm{H}) ;{ }^{13} \mathrm{C} \mathrm{NMR}\left(126 \mathrm{MHz}, \mathrm{CDCl}_{3}\right)$ $\delta 142.2,141.6,128.7,128.4,128.2,127.7,126.7,126.3,76.1,75.2,72.3,71.7,59.1,58.9$, 
57.8, 26.7, 15.6, 14.2; IR (thin film, $\mathrm{NaCl}$ ) 3392, 2984, 2934, 1646, 1452, 1408, 1317, 1199 , 1109, 1050, 1024, $761 \mathrm{~cm}^{-1}$; LRMS (EI, Na) calcd for $\mathrm{C}_{13} \mathrm{H}_{19} \mathrm{NO}_{3} \mathrm{Na}, 260.14 \mathrm{~m} / z(\mathrm{M}+\mathrm{Na})$; observed, $260.27(\mathrm{M}+\mathrm{Na})^{+} \mathrm{m} / z$.

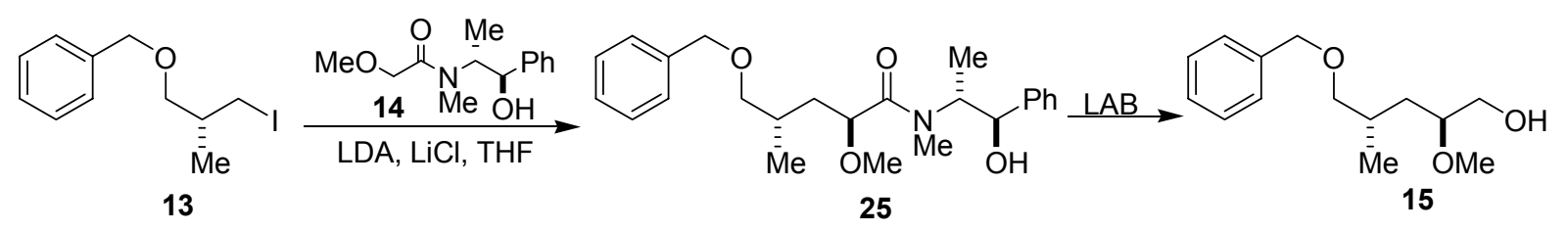

Synthesis of (2S,4S)-5-(benzyloxy)-2-methoxy-4-methylpentan-1-ol, 15. A $250 \mathrm{~mL}$ flask containing lithium chloride $(6.5 \mathrm{~g}, 155 \mathrm{mmol})$ was flame-dried and to it was added diisopropylamine $(7.38 \mathrm{~mL}, 52.6 \mathrm{mmol})$ and THF $(110 \mathrm{~mL})$. The suspension was cooled to $-78{ }^{\circ} \mathrm{C}$, and to it was added $n$-butyllithium in hexanes $(2.5 \mathrm{M}, 19.5 \mathrm{~mL}, 48.8 \mathrm{mmol})$. The resulting solution was warmed to $0{ }^{\circ} \mathrm{C}$ briefly and then was cooled to $-78{ }^{\circ} \mathrm{C}$. Amide $\mathbf{1 4}$ (5.8 g, $24.4 \mathrm{mmol}$ ) was added, and the solution was stirred at $-78{ }^{\circ} \mathrm{C}$ for 1 hour, at $0{ }^{\circ} \mathrm{C}$ for $15 \mathrm{~min}$, and at $23{ }^{\circ} \mathrm{C}$ for $5 \mathrm{~min}$. The mixture was cooled to $0{ }^{\circ} \mathrm{C}$, and iodide $13(4.00 \mathrm{~g}, 13.78 \mathrm{mmol})$ was added to the reaction via syringe as a solution in $10 \mathrm{~mL}$ of THF. The reaction was stirred for 24 hours at $0{ }^{\circ} \mathrm{C}$ and was quenched with half-saturated aqueous ammonium chloride solution $(100 \mathrm{~mL})$. The resulting mixture was extracted with ethyl acetate $(3 \times 50 \mathrm{~mL})$, and the organic layer was then dried over sodium sulfate. The solution was filtered through a pad of silica gel, rinsing with ethyl acetate $(200 \mathrm{~mL})$. All volatiles were removed in vacuo, and the resulting oil $\mathbf{2 5}$ was used in the following reaction. A flame-dried $250 \mathrm{~mL}$ flask was charged with $80 \mathrm{~mL}$ of THF and $8.1 \mathrm{~mL}$ of diisopropylamine $(57.8 \mathrm{mmol})$. This solution was cooled to $-78{ }^{\circ} \mathrm{C}$ and $21.5 \mathrm{~mL}$ of $n$-butyllithium $(2.5 \mathrm{M}$ in hexanes, $53.7 \mathrm{mmol})$ was added dropwise via syringe. The resulting solution was warmed to $0{ }^{\circ} \mathrm{C}$ and stirred for $10 \mathrm{~min}$ before the 
addition of borane-ammonia complex $(90 \%, 1.89 \mathrm{~g}, 55.12 \mathrm{mmol})$. The resulting suspension was stirred at $0{ }^{\circ} \mathrm{C}$ for $15 \mathrm{~min}$ and at $23{ }^{\circ} \mathrm{C}$ for $15 \mathrm{~min}$. The resulting white suspension was cooled to $0{ }^{\circ} \mathrm{C}$ and the crude amide $\mathbf{2 5}$ was added via syringe as a solution in $10 \mathrm{~mL}$ of THF. The solution was allowed to warm to $23{ }^{\circ} \mathrm{C}$, and was stirred for 2 hours. The reaction was slowly quenched with sat. ammonium chloride solution $(50 \mathrm{~mL})$ and the aqueous layer was extracted with $\mathrm{Et}_{2} \mathrm{O}(3 \times 40 \mathrm{~mL})$. The combined organic layers were washed with saturated sodium bicarbonate solution $(1 \times 100 \mathrm{~mL})$ and brine $(1 \times 100 \mathrm{~mL})$ before being dried over sodium sulfate. The solution was filtered, and the solvent was removed in vacuo. The crude product was purified by flash column chromatography (30\% ethyl acetate-hexanes) affording the colorless oil $15(2.29 \mathrm{~g}, 9.65 \mathrm{mmol}, 70 \%$ over 2 steps $)$ as a 9:1 mixture of diastereomers.

Data for (2S,4S)-5-(benzyloxy)-2-methoxy-4-methylpentan-1-ol, 15. $[\alpha]_{589}{ }^{20}+6.19^{\circ}(c$ 0.63, $\left.\mathrm{CHCl}_{3}\right) ;{ }^{1} \mathrm{H} \mathrm{NMR}\left(500 \mathrm{MHz}, \mathrm{CDCl}_{3}\right) \delta$ 7.35-7.26 (m, 5H), 4.50 (app. s, 2H), 3.71 (ddd, $J=11.3,6.1,3.6 \mathrm{~Hz}, 1 \mathrm{H}), 3.48(\mathrm{ddd}, J=11.6,5.8,5.8 \mathrm{~Hz}, 1 \mathrm{H}), 3.41-3.37(\mathrm{~m}, 1 \mathrm{H}), 3.39(\mathrm{~s}$, 3H), 3.34-3.27 (m, 2H), 1.98-1.91 (m, 2H), 1.74 (ddd, $J=14.2,7.4,5.5 \mathrm{~Hz}, 1 \mathrm{H}), 1.22$ (ddd, $J$ $=13.9,8.1,5.2 \mathrm{~Hz}, 1 \mathrm{H}), 0.98(\mathrm{~d}, J=6.8 \mathrm{~Hz}, 3 \mathrm{H}) ;{ }^{13} \mathrm{C} \mathrm{NMR}\left(126 \mathrm{MHz}, \mathrm{CDCl}_{3}\right) \delta 138.6$, $128.3,127.5,127.4,79.5,75.9,73.0,64.0,57.0,35.0,30.0,17.5$; IR (thin film, NaCl) 3447 , 2930, 1457, 1363, 1204, 1099, 737, 698, $668 \mathrm{~cm}^{-1}$; LRMS (EI, Na) calcd for $\mathrm{C}_{14} \mathrm{H}_{22} \mathrm{O}_{3} \mathrm{Na}$, $261.16 \mathrm{~m} / z(\mathrm{M}+\mathrm{Na})$; observed, $261.18(\mathrm{M}+\mathrm{Na})^{+} \mathrm{m} / z$. 


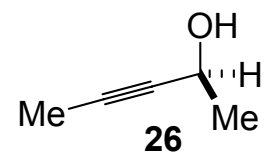

$$
\text { 1) } \mathrm{MsCl}_{1} \mathrm{NEt}_{3}
$$

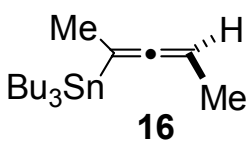

Synthesis of $M$-(-)-2-(Tributylstannyl)-2,3-pentadiene, 16 . To a mixture of 3-pentyn-2-ol $26(2.00 \mathrm{~g}, 23.77 \mathrm{mmol})$ and $\mathrm{NEt}_{3}(6.6 \mathrm{~mL}, 47.54 \mathrm{mmol})$ in $120 \mathrm{~mL}$ of $\mathrm{CH}_{2} \mathrm{Cl}_{2}$, was added methanesulfonyl chloride $(2.78 \mathrm{~mL}, 35.65 \mathrm{mmol})$ at $0{ }^{\circ} \mathrm{C}$. The resulting mixture was stirred at $0{ }^{\circ} \mathrm{C}$ for $1 \mathrm{~h}$, then quenched with saturated $\mathrm{NaHCO}_{3}(100 \mathrm{~mL})$, and extracted with ether $(3 \times$ $50 \mathrm{~mL})$. The ether layer was washed with brine $(1 \times 200 \mathrm{~mL})$ and dried over $\mathrm{Na}_{2} \mathrm{SO}_{4}$. Concentration yielded crude mesylate, which was dried in vacuo and directly used for the next reaction without further purification. To a flame-dried flask charged with diisopropylamine (3.66 mL, $26.2 \mathrm{mmol})$ and THF $(150 \mathrm{~mL})$ was added $n$-BuLi $(2.5 \mathrm{M}$ in hexanes, $9.5 \mathrm{~mL}$, $23.77 \mathrm{mmol})$ at $0^{\circ} \mathrm{C}$. After $30 \mathrm{~min}$, tributyltin hydride $(5.98 \mathrm{~mL}, 22.6 \mathrm{mmol})$ was added dropwise, and the mixture was stirred an additional $30 \mathrm{~min}$. The yellow solution was then cooled to $-78^{\circ} \mathrm{C}$ and $\mathrm{CuBr} \cdot \mathrm{SMe}_{2}(4.64 \mathrm{~g}, 22.6 \mathrm{mmol})$ was added portion-wise. Once addition was complete, the dark solution is stirred an additional $30 \mathrm{~min}$ before the corresponding mesylate of $\mathbf{2 6}$ was added. After $10 \mathrm{~min}$, the solution was poured into a rapidly stirred solution of $500 \mathrm{~mL}$ of 9:1 sat. aq. $\mathrm{NH}_{4} \mathrm{Cl} / \mathrm{NH}_{4} \mathrm{OH}$ solution and $300 \mathrm{~mL}$ of ether. Once the ether layer clarified, it was separated, dried over $\mathrm{MgSO}_{4}$, filtered, and concentrated in vacuo. The resulting oil was purified by chromatography using basic alumina and eluting with hexanes. The recovered stannane $\mathbf{1 6}$ (6.05 g, 71\% over 2 steps) was obtained as a clear and colorless oil, and was of sufficient purity for use in the subsequent propargylation.

Data for $M$-(-)-2-(Tributylstannyl)-2,3-pentadiene, 16: $[\alpha]_{589}{ }^{20}-50.0^{\circ}\left(c=1.29, \mathrm{CHCl}_{3}\right)$; ${ }^{1} \mathrm{H}$ NMR (400 MHz, $\left.\mathrm{CDCl}_{3}\right) \delta 4.59-4.51(\mathrm{~m}, 1 \mathrm{H}), 1.81$ (app. d, $\left.J=3.1 \mathrm{~Hz}, 3 \mathrm{H}\right), 1.61$ (d, $J=$ $6.9 \mathrm{~Hz}, 3 \mathrm{H}), 1.59-1.45(\mathrm{~m}, 6 \mathrm{H}), 1.39-1.27$ (m, 6H), 1.04-0.87 (m, 15H); ${ }^{13} \mathrm{C}$ NMR (100 MHz, 
$\left.\mathrm{CDCl}_{3}\right) \delta 203.7,87.4,75.5,29.2,27.6,19.2,14.3,14.0,9.8$; IR (thin film, $\left.\mathrm{NaCl}\right) 2957,2926$, 2872, 2855, 1943, 1733, 1465, 1457, 1377, 1071, $972 \mathrm{~cm}^{-1}$; LRMS (EI, Na) calcd for $\mathrm{C}_{17} \mathrm{H}_{34} \mathrm{SnH}, 359.17 \mathrm{~m} / \mathrm{z}(\mathrm{M}+\mathrm{H})$; observed, $359.24(\mathrm{M}+\mathrm{H})^{+} \mathrm{m} / z$.

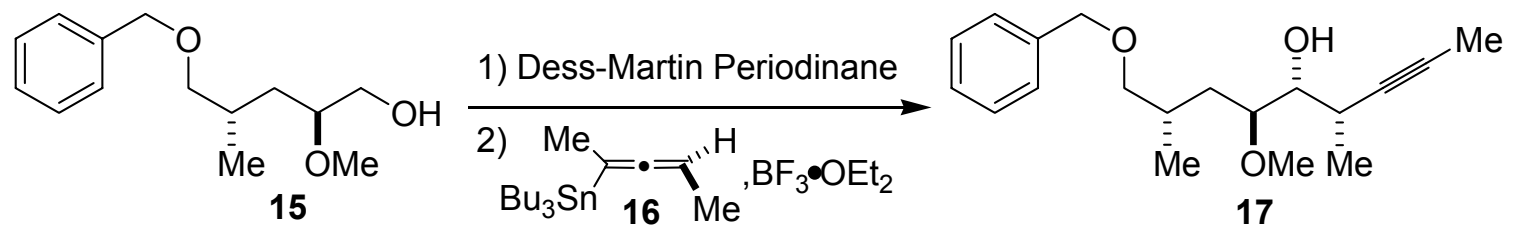

Synthesis of $(2 S, 4 S, 5 R, 6 S)$-1-benzyloxy-4-methoxy-2,6-dimethylnon-7-yn-5-ol, 17: Tо a stirring solution of $15(110 \mathrm{mg}, 0.461 \mathrm{mmol})$ in dichloromethane (1.0 mL, reagent grade) was added 1,1,1-triacetoxy-1,1-dihydro-1,2-benziodoxol-3-(1H)-one (347 $\mathrm{mg}, 0.629 \mathrm{mmol})$ at room temperature. After $1 \mathrm{~h}$ of stirring, the reaction was diluted with a solution of $20 \%$ EtOAc/ hexanes $(10 \mathrm{~mL})$ and most of the solvent was removed. The residue was dissolved in $10 \mathrm{~mL}$ of EtOAc/ hexanes and washed with a $1: 1$ mixture of $10 \% \mathrm{Na}_{2} \mathrm{~S}_{2} \mathrm{O}_{3}$ and sat. $\mathrm{NaHCO}_{3}$ until a clear organic layer was obtained. The organic layer was then washed with brine $(1 \times 10$ $\mathrm{mL}$ ) and dried over anhydrous $\mathrm{Na}_{2} \mathrm{SO}_{4}$. The solution was then filtered and its solvent was removed in vacuo affording the corresponding aldehyde (107 $\mathrm{mg}, 0.454 \mathrm{mmol})$ as a yellow oil. The oil was further dried via azeotropic removal of water (concentrated from anhydrous benzene $(3 \times 3 \mathrm{~mL}))$. The aldehyde was then used in the subsequent step without further purification. To a flame-dried $10 \mathrm{~mL}$ flask containing stannane 16 (324 mg, $0.908 \mathrm{mmol})$ in $1.0 \mathrm{~mL}$ of dichloromethane at $-78{ }^{\circ} \mathrm{C}$ was added the aldehyde from the previous reaction $(107$ $\mathrm{mg}, 0.454 \mathrm{mmol})$ in $1.0 \mathrm{~mL}$ of dichloromethane. $\mathrm{BF}_{3} \cdot \mathrm{OEt}_{2}(57 \mu \mathrm{L}, 0.91 \mathrm{mmol})$ was added slowly via syringe and the solution was stirred at $-78{ }^{\circ} \mathrm{C}$ for $30 \mathrm{~min}$. To the solution was added sat. aq. sodium bicarbonate $(5 \mathrm{~mL})$ and the mixture was warmed to room temperature. 
The aqueous layer was extracted with $\mathrm{Et}_{2} \mathrm{O}(3 \times 5 \mathrm{~mL})$ and the combined organic layers were dried over sodium sulfate, filtered, and the volatiles were removed in vacuo. The crude product was purified using flash column chromatography (10\% ethyl acetate-hexanes) affording a single diastereomer of homopropargylic alcohol 17 (70 $\mathrm{mg}, 0.23 \mathrm{mmol}, 50 \%$ over 2 steps, ds: 4:1)

$(2 S, 4 S, 5 R, 6 S)-1-b e n z y l o x y-4-m e t h o x y-2,6-d i m e t h y l n o n-7-y n-5-o l, ~ 17: ~[\alpha]_{589}{ }^{20}-29.4^{\circ}(c$ 0.50, $\left.\mathrm{CHCl}_{3}\right) ;{ }^{1} \mathrm{H} \mathrm{NMR}\left(400 \mathrm{MHz}, \mathrm{CDCl}_{3}\right) \delta$ 7.37-7.26 (m, $\left.5 \mathrm{H}\right), 4.53(\mathrm{~A}$ of $\mathrm{AB}, J=12.6 \mathrm{~Hz}$, 1H), $4.52(\mathrm{~B}$ of AB, $J=12.6 \mathrm{~Hz}, 1 \mathrm{H}), 3.72-3.68,(\mathrm{~m}, 1 \mathrm{H}), 3.59$ (ddd, $J=10.3,2.6,2.6 \mathrm{~Hz}$, 1H), 3.39 (s, 3H), 3.35 (dd, $J=9.0,6.1 \mathrm{~Hz}, 1 \mathrm{H}), 3.28(\mathrm{dd}, J=9.0,7.1 \mathrm{~Hz}, 1 \mathrm{H}), 2.43-2.35$ (m, 1H), $2.31(\mathrm{~d}, J=1.6 \mathrm{~Hz}, 1 \mathrm{H}), 2.08-2.00(\mathrm{~m}, 1 \mathrm{H}), 1.78(\mathrm{~d}, J=2.3 \mathrm{~Hz}, 3 \mathrm{H}), 1.66$ (ddd, $J=13.9$, $10.3,3.2 \mathrm{~Hz}, 1 \mathrm{H}), 1.27(\mathrm{~d}, J=6.8 \mathrm{~Hz}, 3 \mathrm{H}), 1.25-1.20(\mathrm{~m}, 1 \mathrm{H}), 0.99(\mathrm{~d}, J=6.8 \mathrm{~Hz}, 3 \mathrm{H}) ;{ }^{13} \mathrm{C}$ $\operatorname{NMR}\left(126 \mathrm{MHz}, \mathrm{CDCl}_{3}\right) \delta 138.8,128.3,127.6,127.4,80.1,79.9,77.8,76.5,73.5,73.0,57.1$, 31.5, 29.8, 28.8, 18.4, 16.7, 3.4; IR (thin film, $\mathrm{NaCl}$ ) 3447, 2919, 1496, 1457, 1363, 1260 , 1092, 1029, 928, 802, 736, $698 \mathrm{~cm}^{-1}$; LRMS (EI, Na) calcd for $\mathrm{C}_{19} \mathrm{H}_{28} \mathrm{O}_{3} \mathrm{Na}, 327.20 \mathrm{~m} / z(\mathrm{M}+$ $\mathrm{Na})$; observed, $327.30(\mathrm{M}+\mathrm{Na})^{+} \mathrm{m} / z$.

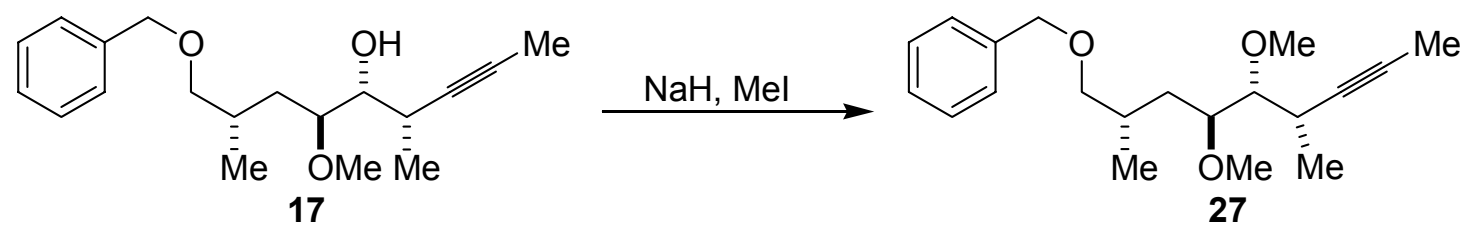

Synthesis of $(2 S, 4 S, 5 R, 6 S)$-1-benzyloxy-4,5-dimethoxy-2,6-dimethyl-7-nonyne, 27: To a flame-dried $10 \mathrm{~mL}$ flask containing a suspension of $\mathrm{NaH}(60 \%, 53 \mathrm{mg}, 1.3 \mathrm{mmol})$ in THF $(1.0 \mathrm{~mL})$ was added homopropargylic alcohol $17(200 \mathrm{mg}, 0.66 \mathrm{mmol})$ via syringe as a solution in THF $(1.0 \mathrm{~mL})$ at $0{ }^{\circ} \mathrm{C}$. After $30 \mathrm{~min}$, methyl iodide $(164 \mu \mathrm{L}, 2.62 \mathrm{mmol})$ was 
added via syringe and the solution was warmed to $23{ }^{\circ} \mathrm{C}$ and held at this temperature for $1 \mathrm{~h}$. The solution was quenched slowly with saturated ammonium chloride solution $(5 \mathrm{~mL})$ and the aqueous layer was extracted with $\mathrm{Et}_{2} \mathrm{O}(3 \times 5 \mathrm{~mL})$. The combined organic layers were dried over sodium sulfate, filtered, and the volatiles were removed in vacuo. The crude product was purified using flash column chromatography (10\% ethyl acetate-hexanes) affording the corresponding methyl ether 27 (205 mg, $0.64 \mathrm{mmol}, 98 \%$ ) as a colorless oil.

(2S,4S,5R,6S)-1-benzyloxy-4,5-dimethoxy-2,6-dimethyl-7-nonyne, 27: $[\alpha]_{589}{ }^{20}-5.45^{\circ}(c$ 0.88, $\left.\mathrm{CHCl}_{3}\right) ;{ }^{1} \mathrm{H} \mathrm{NMR}\left(500 \mathrm{MHz}, \mathrm{CDCl}_{3}\right) \delta$ 7.36-7.26 (m, 5H), $4.51(\mathrm{~A}$ of $\mathrm{AB}, J=12.3 \mathrm{~Hz}$, 1H), $4.50(\mathrm{~B}$ of $\mathrm{AB}, J=12.3 \mathrm{~Hz}, 1 \mathrm{H}), 3.64(\mathrm{ddd}, J=10.0,2.3,2.3 \mathrm{~Hz}, 1 \mathrm{H}), 3.51(\mathrm{~s}, 3 \mathrm{H})$, 3.41-3.38 (m, 1H), $3.39(\mathrm{~s}, 3 \mathrm{H}), 3.27-3.23(\mathrm{~m}, 2 \mathrm{H}), 2.44-2.37,(\mathrm{~m}, 1 \mathrm{H}), 2.08-2.00(\mathrm{~m}, 1 \mathrm{H})$, $1.78(\mathrm{~d}, J=2.6 \mathrm{~Hz}, 3 \mathrm{H}), 1.67(\mathrm{ddd}, J=14.2,10.3,3.6 \mathrm{~Hz}, 1 \mathrm{H}), 1.25-1.19(\mathrm{~m}, 1 \mathrm{H}), 1.22(\mathrm{~d}, J$ $=6.8 \mathrm{~Hz}, 3 \mathrm{H}), 0.99(\mathrm{~d}, J=6.5 \mathrm{~Hz}, 3 \mathrm{H}) ;{ }^{13} \mathrm{C} \mathrm{NMR}\left(126 \mathrm{MHz}, \mathrm{CDCl}_{3}\right) \delta 138.9,128.3,127.5$, $127.3,84.3,80.9,80.8,77.2,76.5,72.9,60.6,57.2,33.0,30.1,28.6,18.2,16.8,3.5$; IR (thin film, $\mathrm{NaCl}) 2931,1454,1363,1196,1099,1028,735,698 \mathrm{~cm}^{-1}$; LRMS (EI, Na) calcd for $\mathrm{C}_{20} \mathrm{H}_{30} \mathrm{O}_{3} \mathrm{Na}, 341.22 \mathrm{~m} / z(\mathrm{M}+\mathrm{Na})$; observed, $341.35(\mathrm{M}+\mathrm{Na})^{+} \mathrm{m} / z$.

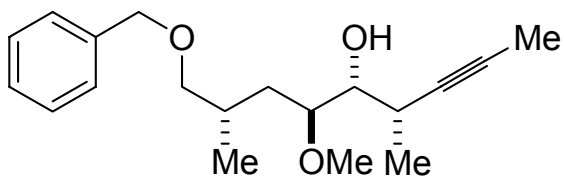

17

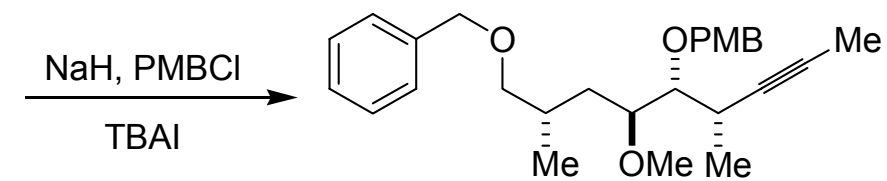

28

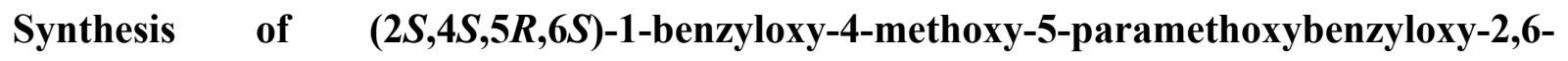
dimethyl-7-nonyne, 28: To a flame-dried $10 \mathrm{~mL}$ flask containing a suspension of $\mathrm{NaH}(60 \%$, $40 \mathrm{mg}, 0.99 \mathrm{mmol})$ in DMF (0.6 mL) was added homopropargylic alcohol 17 (200 mg, 0.66 mmol) as a solution in DMF $(1.0 \mathrm{~mL})$ via syringe at $23{ }^{\circ} \mathrm{C}$. After $20 \mathrm{~min}, p$-methoxybenzyl 
chloride $(180 \mu \mathrm{L}, 1.31 \mathrm{mmol})$ was added via syringe followed by the addition of tetrabutylammonium iodide ( $48 \mathrm{mg}, 0.13 \mathrm{mmol}$ ), and the solution was stirred at $23{ }^{\circ} \mathrm{C}$ for 12 hours. The reaction was quenched slowly with sat. ammonium chloride solution $(5 \mathrm{~mL})$ and the aqueous layer was extracted with $\mathrm{Et}_{2} \mathrm{O}(3 \times 5 \mathrm{~mL})$. The combined organic layers were dried over sodium sulfate, filtered, and volatiles removed in vacuo. The crude product was purified using flash column chromatography (15\% ethyl acetate-hexanes) affording the corresponding PMB-protected alcohol 28 (244 mg, $0.58 \mathrm{mmol}, 88 \%)$ as a colorless oil.

$(2 S, 4 S, 5 R, 6 S)$-1-benzyloxy-4-methoxy-5-paramethoxybenzyloxy-2,6-dimethyl-7-nonyne,

28: $[\alpha]_{589}{ }^{20}+19.0^{\circ}\left(c 0.40, \mathrm{CHCl}_{3}\right) ;{ }^{1} \mathrm{H}$ NMR $\left(500 \mathrm{MHz}, \mathrm{CDCl}_{3}\right) \delta$ 7.37-7.26 (m, 7H), 6.88$6.84(\mathrm{~m}, 2 \mathrm{H}), 4.68(\mathrm{~A}$ of $\mathrm{AB}, J=11.0 \mathrm{~Hz}, 1 \mathrm{H}), 4.61(\mathrm{~B}$ of $\mathrm{AB}, J=11.0 \mathrm{~Hz}, 1 \mathrm{H}), 4.53-4.50$ (m, 2H), 3.80 (s, 3H), 3.71 (ddd, $J=10.1,2.2,2.2 \mathrm{~Hz}, 1 \mathrm{H}), 3.51$ (dd, $J=8.8,2.2 \mathrm{~Hz}, 1 \mathrm{H})$, 3.41-3.38 (m, 1H), $3.41(\mathrm{~s}, 3 \mathrm{H}), 3.27(\mathrm{dd}, J=9.1,7.3 \mathrm{~Hz}, 1 \mathrm{H}), 2.48-2.40(\mathrm{~m}, 1 \mathrm{H}), 2.11-2.02$ (m, $1 \mathrm{H}), 1.78(\mathrm{~d}, J=2.5 \mathrm{~Hz}, 3 \mathrm{H}), 1.76(\mathrm{ddd}, J=14.2,10.1,3.5 \mathrm{~Hz}, 1 \mathrm{H}) 1.30-1.23(\mathrm{~m}, 1 \mathrm{H})$, $1.22(\mathrm{~d}, J=6.6 \mathrm{~Hz}, 3 \mathrm{H}), 0.99(\mathrm{~d}, J=6.6 \mathrm{~Hz}, 3 \mathrm{H}) ;{ }^{13} \mathrm{C} \mathrm{NMR}\left(126 \mathrm{MHz}, \mathrm{CDCl}_{3}\right) \delta 159.1$, $138.9,131.0,129.7,128.3,127.5,127.3,113.6,81.4,81.0,77.7,77.2,76.5,73.6,72.9,57.2$ 55.3, 33.3, 30.2, 28.5, 18.4, 16.9, 3.4; IR (thin film, $\mathrm{NaCl}$ ) 2918, 1613, 1514, 1454, 1302, 1249, 1173, 1098, 1036, 822, 751, $698 \mathrm{~cm}^{-1}$; LRMS (EI, Na) calcd for $\mathrm{C}_{27} \mathrm{H}_{36} \mathrm{O}_{4} \mathrm{Na}, 447.26$ $m / z(\mathrm{M}+\mathrm{Na})$; observed, $447.34(\mathrm{M}+\mathrm{Na})^{+} m / z$. 


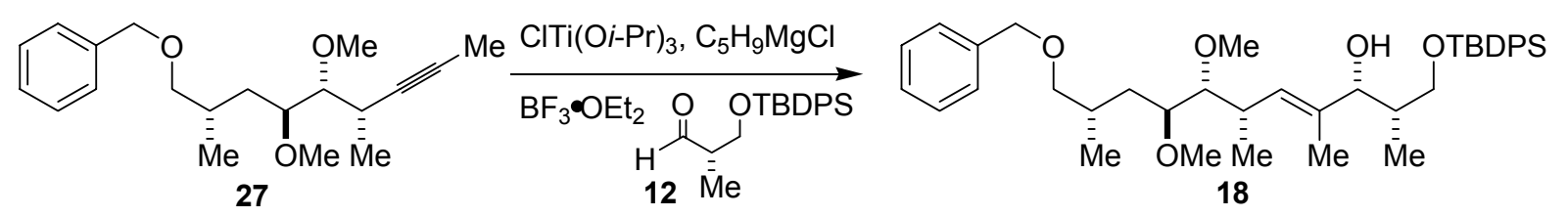

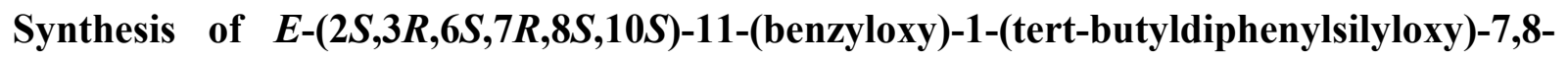
dimethoxy-2,4,6,10-tetramethylundec-4-en-3-ol, 18: To a $-78^{\circ} \mathrm{C}$ solution of alkyne 27 (50 $\mathrm{mg}, 0.16 \mathrm{mmol})$ in $1.5 \mathrm{~mL}$ of toluene, was added $314 \mu \mathrm{L}$ of $\mathrm{ClTi}(\mathrm{O} i-\mathrm{Pr})_{3}(1.0 \mathrm{M}$ in hexanes; $0.314 \mathrm{mmol})$ and $314 \mu \mathrm{L}$ of $c-\mathrm{C}_{5} \mathrm{H}_{9} \mathrm{MgCl}(2.0 \mathrm{M}$ in diethyl ether; $0.628 \mathrm{mmol})$ in a dropwise manner via a dry gas-tight syringe. The resulting yellow solution was slowly warmed to -30 ${ }^{\circ} \mathrm{C}$, stirred for $1 \mathrm{~h}$, then cooled to $-78{ }^{\circ} \mathrm{C}$ before adding $\mathrm{BF}_{3} \cdot \mathrm{OEt}_{2}(59 \mu \mathrm{L}, 0.47 \mathrm{mmol})$ dropwise followed by the addition of aldehyde $11(158 \mathrm{mg}, 0.471 \mathrm{mmol})$ as a solution in 400 $\mu \mathrm{L}$ of toluene. After stirring for $1 \mathrm{~h}$, the reaction was quenched at $-78^{\circ} \mathrm{C}$ with $1.0 \mathrm{~mL}$ of sat. $\mathrm{NH}_{4} \mathrm{Cl}$ solution. This solution was warmed to ambient temperature before partitioning between EtOAc $(10 \mathrm{~mL})$ and water $(10 \mathrm{~mL})$. The aqueous layer was extracted with EtOAc (3 $\times 5 \mathrm{~mL})$ and the combined organic layer was then washed with sat. $\mathrm{NaHCO}_{3}$ solution $(2 \times 15$ $\mathrm{mL})$ and brine $(1 \times 15 \mathrm{~mL})$, before being dried over $\mathrm{Na}_{2} \mathrm{SO}_{4}$. The crude material $(\mathrm{dr}: 2.5: 1)$ was purified by flash column chromatography on silica gel eluting with $10 \%$ EtOAc-hexanes to provide 18 as a single diastereomer ( $57 \mathrm{mg}, 0.088 \mathrm{mmol}, 54 \%$ yield).

Data for major diastereomer, $E-(2 S, 3 R, 6 S, 7 R, 8 S, 10 S)-11-(b e n z y l o x y)-1-($ tertbutyldiphenylsilyloxy)-7,8-dimethoxy-2,4,6,10-tetramethylundec-4-en-3-ol, $18:[\alpha]_{589}{ }^{20}-$ $1.3^{\circ}\left(c=0.47, \mathrm{CHCl}_{3}\right) ;{ }^{1} \mathrm{H} \mathrm{NMR}\left(500 \mathrm{MHz}, \mathrm{CDCl}_{3}\right) \delta$ 7.69-7.65 (m, 4H), 7.47-7.26 (m, $11 \mathrm{H}), 5.32(\mathrm{~d}, J=10.4 \mathrm{~Hz}, 1 \mathrm{H}), 4.50(\mathrm{~A}$ of $\mathrm{AB}, J=12.3 \mathrm{~Hz}, 1 \mathrm{H}), 4.48(\mathrm{~B}$ of $\mathrm{AB}, J=12.3 \mathrm{~Hz}$ 1H); 4.22 (app. s, 1H), 3.75-3.66 (m, 2H), $3.47(\mathrm{~s}, 3 \mathrm{H}), 3.38(\mathrm{dd}, J=9.1,5.4 \mathrm{~Hz}, 1 \mathrm{H}), 3.33$ (s, $3 \mathrm{H}), 3.27-3.20(\mathrm{~m}, 2 \mathrm{H}), 3.16(\mathrm{dd}, J=8.5,2.2 \mathrm{~Hz}, 1 \mathrm{H}), 2.55(\mathrm{~d}, J=3.2 \mathrm{~Hz}, 1 \mathrm{H}), 2.52-2.43(\mathrm{~m}$, 
1H), 2.02-1.93 (m, 1H), 1.88-1.81 (m, 1H), $1.66(\mathrm{ddd}, J=14.2,10.4,3.8 \mathrm{~Hz}, 1 \mathrm{H}), 1.52$ (d, $J=$ $0.6 \mathrm{~Hz}, 3 \mathrm{H}), 1.12$ (ddd, $J=14.5,10.4,2.2 \mathrm{~Hz}, 1 \mathrm{H}), 1.07$ (s, 9H), 1.03 (d, $J=6.6 \mathrm{~Hz}, 3 \mathrm{H})$, $0.89(\mathrm{~d}, J=6.6 \mathrm{~Hz}, 3 \mathrm{H}), 0.82(\mathrm{~d}, J=6.9 \mathrm{~Hz}, 3 \mathrm{H}) ;{ }^{13} \mathrm{C} \mathrm{NMR}\left(126 \mathrm{MHz}, \mathrm{CDCl}_{3}\right) \delta 138.9$, $135.7,135.6,134.7,133.3,133.2,129.8,129.7,128.3,128.2,127.7,127.6,127.5,127.3,85.2$, 81.0, 77.2, 76.9, 76.6, 72.9, 68.3, 60.8, 57.2, 38.0, 34.9, 33.2, 30.1, 26.9, 19.2, 17.6, 16.9, 14.0, 9.7; IR (thin film, NaCl) 3476, 2958, 2928, 2856, 1473, 1462, 1428, 1113, 1105, 1021

$\mathrm{cm}^{-1}$; LRMS (EI, Na) calcd for $\mathrm{C}_{40} \mathrm{H}_{58} \mathrm{O}_{5} \mathrm{SiNa}, 669.41 \mathrm{~m} / z(\mathrm{M}+\mathrm{Na})$; observed, $669.71(\mathrm{M}+$ $\mathrm{Na})^{+} m / z$.

Data for Minor Diastereomer, $E-(2 S, 3 S, 6 S, 7 R, 8 S, 10 S)-11-(b e n z y l o x y)-1-(t e r t-$ butyldiphenylsilyloxy)-7,8-dimethoxy-2,4,6,10-tetramethylundec-4-en-3-ol, $18 \mathrm{~b}:[\alpha]_{589}{ }^{20}$ $+5.6^{\circ}\left(c=0.36, \mathrm{CHCl}_{3}\right) ;{ }^{1} \mathrm{H} \operatorname{NMR}\left(500 \mathrm{MHz}, \mathrm{CDCl}_{3}\right) \delta$ 7.70-7.67 (m, 4H), 7.47-7.36 (m, 7H), 7.34-7.30 (m, 4H), $5.20(\mathrm{~d}, J=10.0 \mathrm{~Hz}, 1 \mathrm{H}), 4.50(\mathrm{~A}$ of AB, $J=12.3 \mathrm{~Hz}, 1 \mathrm{H}), 4.49$ (B of $\mathrm{AB}, J=12.3 \mathrm{~Hz}, 1 \mathrm{H}), 3.88$, (dd, $J=8.8,0.6 \mathrm{~Hz}, 1 \mathrm{H}), 3.77(\mathrm{dd}, J=10.1,4.1 \mathrm{~Hz}, 1 \mathrm{H}), 3.68$ (dd, $J=10.1,7.9,1 \mathrm{H}), 3.61(\mathrm{~d}, J=1.9 \mathrm{~Hz}, 1 \mathrm{H}), 3.49(\mathrm{~s}, 3 \mathrm{H}), 3.38(\mathrm{dd}, J=9.1,5.7 \mathrm{~Hz}, 1 \mathrm{H})$, 3.35 (s, 3H), 3.31 (ddd, $J=10.4,2.8,2.8 \mathrm{~Hz}, 1 \mathrm{H}), 3.23$ (dd, $J=9.1,7.3,1 \mathrm{H}), 3.16$ (dd, $J=$ 7.9, $2.5 \mathrm{~Hz}, 1 \mathrm{H}), 2.53-2.45(\mathrm{~m}, 1 \mathrm{H}), 2.04-1.96(\mathrm{~m}, 1 \mathrm{H}), 1.93-1.86(\mathrm{~m}, 1 \mathrm{H}), 1.69$ (ddd, $J=$ 14.5, 10.4, $3.8 \mathrm{~Hz}, 1 \mathrm{H}), 1.62(\mathrm{~d}, J=1.0 \mathrm{~Hz}, 3 \mathrm{H}), 1.16$ (ddd, $J=14.5,10.0,2.2 \mathrm{~Hz}, 1 \mathrm{H}), 1.07$ (s, 9H), $1.00(\mathrm{~d}, J=6.6 \mathrm{~Hz}, 3 \mathrm{H}), 0.93(\mathrm{~d}, J=6.6 \mathrm{~Hz}, 3 \mathrm{H}), 0.62(\mathrm{~d}, J=6.9 \mathrm{~Hz}, 3 \mathrm{H}) ;{ }^{13} \mathrm{C} \mathrm{NMR}$ $\left(126 \mathrm{MHz}, \mathrm{CDCl}_{3}\right) \delta 138.9,135.6,132.9,130.9,129.8,128.2,127.8,127.5,127.3,85.1,83.5$, $80.9,76.5,72.8,69.3,60.8,57.1,38.2,34.6,33.4,30.1,26.8,19.1,17.2,17.0,13.6,11.2$; IR (thin film, NaCl) 3503, 2959, 2928, 2856, 1473, 1472, 1457, 1112, 1029, $1008 \mathrm{~cm}^{-1}$; LRMS (EI, Na) calcd for $\mathrm{C}_{40} \mathrm{H}_{58} \mathrm{O}_{5} \mathrm{SiNa}, 669.41 \mathrm{~m} / z(\mathrm{M}+\mathrm{Na})$; observed, $669.78(\mathrm{M}+\mathrm{Na})^{+} \mathrm{m} / z$. 


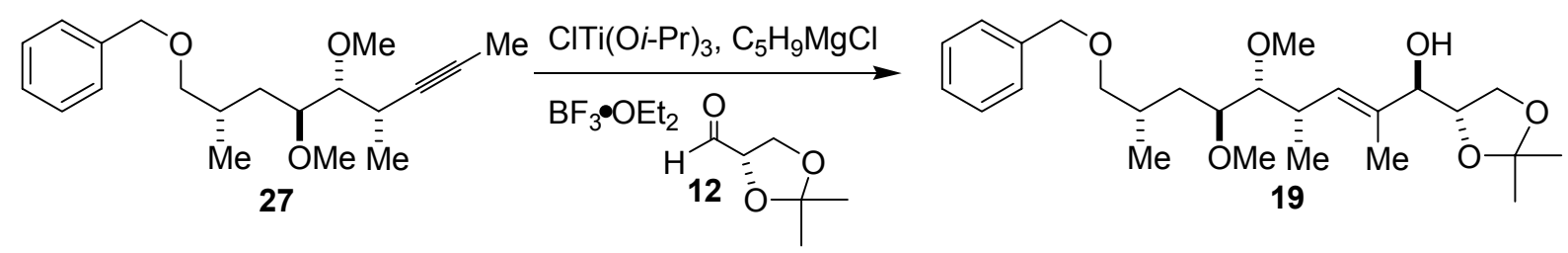

Synthesis of $E-(1 R, 4 S, 5 R, 6 S, 8 S)-9-($ benzyloxy)-1-((S)-2,2-dimethyl-1,3-dioxolan-4-yl)5,6-dimethoxy-2,4,8-trimethylnon-2-en-1-ol: To a $-78{ }^{\circ} \mathrm{C}$ solution of alkyne 27 (50 mg, $0.16 \mathrm{mmol})$ in $1.5 \mathrm{~mL}$ of toluene, was added $314 \mu \mathrm{L}$ of $\mathrm{ClTi}(\mathrm{O} i \text { - } \mathrm{Pr})_{3}(1.0 \mathrm{M}$ in hexanes; 0.314 $\mathrm{mmol})$ and $314 \mu \mathrm{L}$ of $c-\mathrm{C}_{5} \mathrm{H}_{9} \mathrm{MgCl}(2.0 \mathrm{M}$ in diethyl ether; $0.628 \mathrm{mmol})$ in a dropwise manner via a dry gas-tight syringe. The resulting yellow solution was slowly warmed to $-30{ }^{\circ} \mathrm{C}$, stirred for $1 \mathrm{~h}$, then cooled to $-78{ }^{\circ} \mathrm{C}$ before adding $\mathrm{BF}_{3} \cdot \mathrm{OEt}_{2}(59 \mu \mathrm{L}, 0.47 \mathrm{mmol})$ dropwise followed by the addition of aldehyde $\mathbf{1 2}^{1}(61 \mathrm{mg}, 0.47 \mathrm{mmol})$ as a solution in $400 \mu \mathrm{L}$ of toluene. After stirring for $1 \mathrm{~h}$, the reaction was quenched at $-78^{\circ} \mathrm{C}$ with $1.0 \mathrm{~mL}$ of saturated $\mathrm{NH}_{4} \mathrm{Cl}$ solution. This solution was warmed to ambient temperature before partitioning between EtOAc $(10 \mathrm{~mL})$ and water $(10 \mathrm{~mL})$. The aqueous layer was extracted with EtOAc (3 $\times 5 \mathrm{~mL})$. The combined organic layer was then washed with sat. $\mathrm{NaHCO}_{3}$ solution $(2 \times 15$ $\mathrm{mL})$ and brine $(1 \times 15 \mathrm{~mL})$, before being dried over $\mathrm{Na}_{2} \mathrm{SO}_{4}$. The crude material $(\mathrm{dr}: 3: 1)$ was purified by flash column chromatography on silica gel eluting with $20 \%$ EtOAc-hexanes to provide a diastereomeric mixture of compounds 19 and $\mathbf{2 0}$ (41 $\mathrm{mg}, 0.091 \mathrm{mmol}, 57 \%$ yield). The diastereomers were then separated by HPLC for characterization [EtOAc/ hexanes: $10 \%$ (0-5 min, $20 \mathrm{~mL} / \mathrm{min}), 10-18 \%(5-10 \mathrm{~min}, 20 \mathrm{~mL} / \mathrm{min}), 18 \%(10-20 \mathrm{~min}, 20 \mathrm{~mL} / \mathrm{min}), 18-$ $25 \%$ (20-26 min, $20 \mathrm{~mL} / \mathrm{min}), 25 \%$ (26-34 $\mathrm{min}, 20 \mathrm{~mL} / \mathrm{min})]$.

Data for $E-(1 R, 4 S, 5 R, 6 S, 8 S)-9-($ benzyloxy)-1-((S)-2,2-dimethyl-1,3-dioxolan-4-yl)-5,6dimethoxy-2,4,8-trimethylnon-2-en-1-ol, 19: $[\alpha]_{589}{ }^{20}-6.4{ }^{\circ}\left(c=0.39, \mathrm{CHCl}_{3}\right) ;{ }^{1} \mathrm{H} \mathrm{NMR}$ $\left(500 \mathrm{MHz}, \mathrm{CDCl}_{3}\right) \delta 7.34-7.26(\mathrm{~m}, 5 \mathrm{H}), 5.42(\mathrm{~d}, J=10.1 \mathrm{~Hz}, 1 \mathrm{H}), 4.50(\mathrm{~A}$ of $\mathrm{AB}, J=12.6$ 
$\mathrm{Hz}, 1 \mathrm{H}), 4.49$ (B of AB, $J=12.6 \mathrm{~Hz}, 1 \mathrm{H}), 4.20$ (ddd, $J=6.6,6.6,4.4 \mathrm{~Hz}, 1 \mathrm{H}), 4.17-4.14$ (m, 1H), 3.89 (app. d, $J=6.6 \mathrm{~Hz}, 2 \mathrm{H}), 3.49$ (s, 3H), 3.38 (dd, $J=9.1,5.4 \mathrm{~Hz}, 1 \mathrm{H}), 3.35$ (s, 3H), $3.23(\mathrm{dd}, J=9.1,7.3 \mathrm{~Hz}, 1 \mathrm{H}), 3.19$ (ddd, $J=10.4,2.2,2.2 \mathrm{~Hz}, 1 \mathrm{H}), 3.15$ (dd, $J=8.5,2.2 \mathrm{~Hz}$, 1H), 2.52-2.45 (m, 1H), 2.09 (d, $J=2.2 \mathrm{~Hz}, 1 \mathrm{H}), 2.03-1.95(\mathrm{~m}, 1 \mathrm{H}), 1.69$ (ddd, $J=14.2,10.4$, $3.8 \mathrm{~Hz}, 1 \mathrm{H}), 1.62(\mathrm{~d}, J=1.3 \mathrm{~Hz}, 3 \mathrm{H}), 1.44(\mathrm{~s}, 3 \mathrm{H}), 1.35$ (s, 3H), 1.12 (ddd, $J=14.2,9.8,1.9$ $\mathrm{Hz}, 1 \mathrm{H}), 1.04(\mathrm{~d}, J=6.6 \mathrm{~Hz}, 3 \mathrm{H}), 0.90(\mathrm{~d}, J=6.6 \mathrm{~Hz}, 3 \mathrm{H}) ;{ }^{13} \mathrm{C} \mathrm{NMR}\left(126 \mathrm{MHz}, \mathrm{CDCl}_{3}\right) \delta$ $138.9,132.4,129.5,128.3,127.6,127.4,109.3,85.0,81.0,77.2,76.5,74.8,73.0,64.7,60.9$, 57.3, 34.7, 33.1, 30.1, 26.6, 25.1, 17.5, 17.0, 14.0; IR (thin film, NaCl) 3447, 2918, 2849, 1457, 1380, 1370, 1245, 1213, 1099, 1069, $854 \mathrm{~cm}^{-1}$; LRMS (EI, Na) calcd for $\mathrm{C}_{26} \mathrm{H}_{42} \mathrm{O}_{6} \mathrm{Na}$, $473.30 \mathrm{~m} / \mathrm{z}(\mathrm{M}+\mathrm{Na})$; observed, $473.54(\mathrm{M}+\mathrm{Na})^{+} \mathrm{m} / \mathrm{z}$.

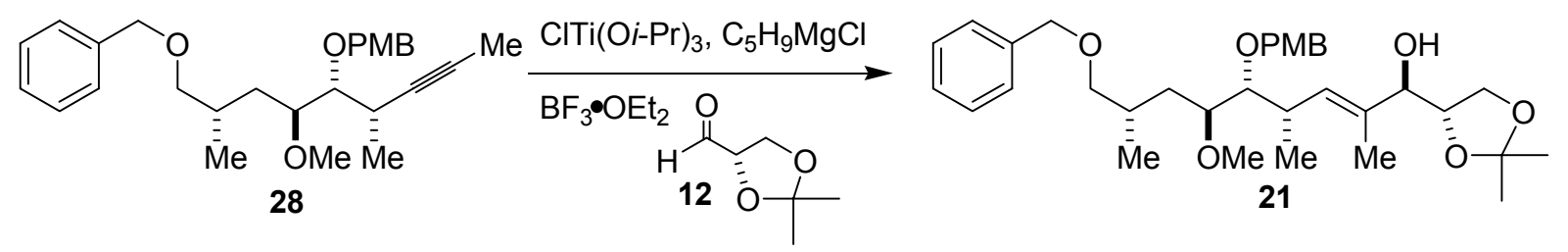

Synthesis of $E-(1 R, 4 S, 5 R, 6 S, 8 S)-5-($ paramethoxybenzyloxy)-9-(benzyloxy)-1-((S)-2,2dimethyl-1,3-dioxolan-4-yl)-6-methoxy-2,4,8-trimethylnon-2-en-1-ol, 21: To a $-78{ }^{\circ} \mathrm{C}$ solution of alkyne $28(10 \mathrm{mg}, 0.024 \mathrm{mmol})$ in $300 \mu \mathrm{L}$ of toluene, was added $48 \mu \mathrm{L}$ of $\mathrm{ClTi}(\mathrm{O} i-\mathrm{Pr})_{3}(1.0 \mathrm{M}$ in hexanes; $0.047 \mathrm{mmol})$ and $48 \mu \mathrm{L}$ of $c-\mathrm{C}_{5} \mathrm{H}_{9} \mathrm{MgCl}(2.0 \mathrm{M}$ in diethyl ether; $0.095 \mathrm{mmol}$ ) in a dropwise manner via a dry gas-tight syringe. The resulting yellow solution was slowly warmed to $-30{ }^{\circ} \mathrm{C}$, stirred for $1 \mathrm{~h}$, then cooled to $-78{ }^{\circ} \mathrm{C}$ before adding $\mathrm{BF}_{3} \cdot \mathrm{OEt}_{2}(9 \mu \mathrm{L}, 0.07 \mathrm{mmol})$ dropwise followed by the addition of aldehyde $\mathbf{1 2}^{1}(10 \mathrm{mg}$, $0.071 \mathrm{mmol}$ ) as a solution in $35 \mu \mathrm{L}$ of toluene. After stirring for $1 \mathrm{~h}$, the reaction was quenched at $-78^{\circ} \mathrm{C}$ with $1.0 \mathrm{~mL}$ of sat. $\mathrm{NH}_{4} \mathrm{Cl}$ solution. This solution was warmed to ambient 
temperature before partitioning between EtOAc $(10 \mathrm{~mL})$ and water $(10 \mathrm{~mL})$. The aqueous layer was extracted with EtOAc $(3 \times 5 \mathrm{~mL})$. The combined organic layer was then washed with sat. $\mathrm{NaHCO}_{3}$ solution $(2 \times 15 \mathrm{~mL})$ and brine $(1 \times 15 \mathrm{~mL})$, before being dried over $\mathrm{Na}_{2} \mathrm{SO}_{4}$. The crude material (dr: 2.5:1) was purified by flash column chromatography on silica gel eluting with 20\% EtOAc-hexanes to provide a diastereomeric mixture of compounds $\mathbf{2 1}$ and $22(8.5 \mathrm{mg}, 0.015 \mathrm{mmol}, 65 \%$ yield $)$. The diastereomers were then separated by HPLC for characterization [EtOAc/ hexanes: $15 \%$ (0-5 $\mathrm{min}, 20 \mathrm{~mL} / \mathrm{min}), 15-20 \%(5-9 \mathrm{~min}, 20 \mathrm{~mL} /$ $\min$ ), 20\% (9-12 $\mathrm{min}, 20 \mathrm{~mL} / \mathrm{min}$ ), $20-30 \%$ (12-20 $\mathrm{min}, 20 \mathrm{~mL} / \mathrm{min}), 30 \%$ (20-23 $\mathrm{min}, 20$ $\mathrm{mL} / \mathrm{min}), 30-50 \%$ (23-25 $\mathrm{min}, 20 \mathrm{~mL} / \mathrm{min}), 50 \%(25-35 \mathrm{~min}, 20 \mathrm{~mL} / \mathrm{min})]$.

Data for $\quad E-(1 R, 4 S, 5 R, 6 S, 8 S)-5-($ paramethoxybenzyloxy)-9-(benzyloxy)-1-((S)-2,2dimethyl-1,3-dioxolan-4-yl)-6-methoxy-2,4,8-trimethylnon-2-en-1-ol, $21::[\alpha]_{589}{ }^{20}+3.5^{\circ}$ $\left(c=0.43, \mathrm{CHCl}_{3}\right) ;{ }^{1} \mathrm{H}$ NMR $\left(500 \mathrm{MHz}, \mathrm{CDCl}_{3}\right) \delta$ 7.35-7.25 (m, 7H), 6.88-6.84 (m, 2H), 5.40 (d, $J=10.0 \mathrm{~Hz}, 1 \mathrm{H}$ ), 4.63 (A of AB, $J=11.0 \mathrm{~Hz}, 1 \mathrm{H}$ ), 4.60 (B of AB, $J=11.0 \mathrm{~Hz}, 1 \mathrm{H}$ ), 4.50 (app. s, 2H), 4.19 (ddd, $J=6.8,6.8,4.5 \mathrm{~Hz}, 1 \mathrm{H}$ ), 4.15-4.13 (m, 1H), 3.89 (app. d, $J=6.5 \mathrm{~Hz}$, 2H), $3.80(\mathrm{~s}, 3 \mathrm{H}), 3.43(\mathrm{dd}, J=9.0,1.6,1 \mathrm{H}), 3.38(\mathrm{dd}, J=9.0,5.5 \mathrm{~Hz}, 1 \mathrm{H}), 3.35(\mathrm{~s}, 3 \mathrm{H})$, 3.27-3.21 (m, 2H), 2.56-2.48 (m, 1H), $2.05(\mathrm{~d}, J=2.6 \mathrm{~Hz}, 1 \mathrm{H}), 2.04-1.98(\mathrm{~m}, 1 \mathrm{H}), 1.79$ (ddd, $J=14.2,10.3,3.6 \mathrm{~Hz}, 1 \mathrm{H}), 1.62$, (d, $J=1.0 \mathrm{~Hz}, 3 \mathrm{H}), 1.44$ (s, 3H), 1.35 (s, 3H), 1.18 (ddd, $J=$ 14.2, 9.7, $1.6 \mathrm{~Hz}, 1 \mathrm{H}), 1.03(\mathrm{~d}, J=6.5 \mathrm{~Hz}, 3 \mathrm{H}), 0.90(\mathrm{~d}, J=6.5 \mathrm{~Hz}, 3 \mathrm{H}) ;{ }^{13} \mathrm{C} \mathrm{NMR}(126$ $\left.\mathrm{MHz}, \mathrm{CDCl}_{3}\right) \delta 159.1,138.8,132.4,131.1,129.7,129.5,128.3,127.6,127.4,113.7,109.3$, $82.0,81.6,77.2,76.5,74.7,73.8,72.9,64.7,57.3,55.3,34.6,33.4,30.1,26.5,25.1,17.9$, 17.1, 14.0; IR (thin film, $\mathrm{NaCl}$ ) 3468, 2957, 2917, 2872, 2850, 1612, 1514, 1454, 1370, 1248, 1098, 1066, 849, $820 \mathrm{~cm}^{-1}$; LRMS (EI, Na) calcd for $\mathrm{C}_{33} \mathrm{H}_{48} \mathrm{O}_{7} \mathrm{Na}, 579.34 \mathrm{~m} / z(\mathrm{M}+\mathrm{Na})$; observed, $579.56(\mathrm{M}+\mathrm{Na})^{+} \mathrm{m} / z$. 


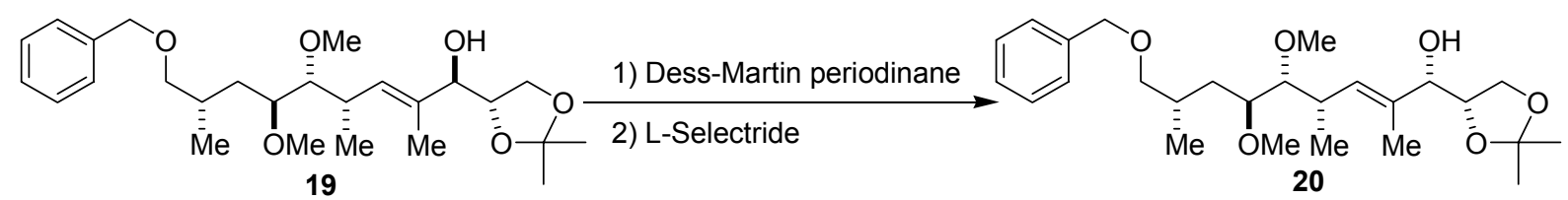

Synthesis of $E$-(1S,4S,5R,6S,8S)-9-(benzyloxy)-1-((S)-2,2-dimethyl-1,3-dioxolan-4-yl)-5,6dimethoxy-2,4,8-trimethylnon-2-en-1-ol, 20: To a stirring solution of a diastereomeric mixture of 19 and 20 (37 mg, $0.082 \mathrm{mmol})$ in dichloromethane $(0.8 \mathrm{~mL}$, reagent grade) was added 1,1,1-triacetoxy-1,1-dihydro-1,2-benziodoxol-3-(1H)-one (52 mg, $0.123 \mathrm{mmol}$ ) at room temperature. After $1 \mathrm{~h}$ of stirring, the reaction was diluted with a solution of $20 \%$ EtOAc/ hexanes $(10 \mathrm{~mL})$ and most of the solvent was removed. The residue was dissolved in $10 \mathrm{~mL}$ of EtOAc/ hexanes and washed with a 1:1 mixture of $10 \% \mathrm{Na}_{2} \mathrm{~S}_{2} \mathrm{O}_{3}$ and sat. $\mathrm{NaHCO}_{3}$ until a clear organic layer was obtained. The organic layer was then washed with brine $(1 \times 10 \mathrm{~mL})$ and dried over anhydrous $\mathrm{Na}_{2} \mathrm{SO}_{4}$. The solvent was removed in vacuo and the residue was purified by flash column chromatography eluting with $20 \%$ EtOAc/ hexanes to afford the corresponding enone which was immediately used in the following step. To a cooled solution of recovered enone in $800 \mu \mathrm{L}$ of $\mathrm{THF}$ at $-78{ }^{\circ} \mathrm{C}$ was added L-Selectride $(1.0 \mathrm{M}$ in hexanes; $246 \mu \mathrm{L}, 0.246 \mathrm{mmol})$ slowly via syringe down the side of the flask. After stirring for $30 \mathrm{~min}$, $0.5 \mathrm{~mL}$ of $\mathrm{H}_{2} \mathrm{O}$ was added and the suspension was allowed to reach room temperature. All contents were loaded directly on a silica gel column, and the product was purified by flash column chromatography eluting with $20 \%$ EtOAc/ hexanes affording $31 \mathrm{mg}$ of unsaturated alcohol 20 (0.069 mmol, dr $\geq 20: 1,84 \%$ over 2 steps).

Data for $E-(1 S, 4 S, 5 R, 6 S, 8 S)-9-(b e n z y l o x y)-1-((S)-2,2-d i m e t h y l-1,3-d i o x o l a n-4-y l)-5,6-$ dimethoxy-2,4,8-trimethylnon-2-en-1-ol, 20: $[\alpha]_{365}{ }^{20}+5.8^{\circ}\left(c=0.59, \mathrm{CHCl}_{3}\right) ;{ }^{1} \mathrm{H} \mathrm{NMR}$ $\left(500 \mathrm{MHz}, \mathrm{CDCl}_{3}\right) \delta$ 7.34-7.26 (m, 5H), $5.32(\mathrm{~d}, J=10.1 \mathrm{~Hz}, 1 \mathrm{H}), 4.49$ (app. s, 2H), 4.13 (dd, $J=13.6,6.3 \mathrm{~Hz}, 1 \mathrm{H}), 3.94(\mathrm{dd}, J=8.2,6.6 \mathrm{~Hz}, 1 \mathrm{H}), 3.90-3.85(\mathrm{~m}, 1 \mathrm{H}), 3.68(\mathrm{dd}, J=8.2,6.0$ 
Hz, 1H), 3.48 (s, 3H), 3.38 (dd, $J=9.1,5.4 \mathrm{~Hz}, 1 \mathrm{H}), 3.35$ (s, 3H), 3.23 (dd, $J=8.8,7.3 \mathrm{~Hz}$, $1 \mathrm{H}), 3.17$ (ddd, $J=10.4,2.2,2.2 \mathrm{~Hz}, 1 \mathrm{H}), 3.12(\mathrm{dd}, J=8.5,2.2 \mathrm{~Hz}, 1 \mathrm{H}), 2.52-2.44(\mathrm{~m}, 1 \mathrm{H})$, $2.41(\mathrm{~d}, J=3.2 \mathrm{~Hz}, 1 \mathrm{H}), 2.04-1.95(\mathrm{~m}, 1 \mathrm{H}), 1.70(\mathrm{ddd}, J=14.2,10.4,3.8 \mathrm{~Hz}, 1 \mathrm{H}), 1.64$ (d, $J$ $=1.3 \mathrm{~Hz}, 3 \mathrm{H}), 1.46(\mathrm{~s}, 3 \mathrm{H}), 1.36(\mathrm{~s}, 3 \mathrm{H}), 1.13(\mathrm{ddd}, J=14.2,10.1,2.2 \mathrm{~Hz}, 1 \mathrm{H}), 1.04(\mathrm{~d}, J=$ $6.6 \mathrm{~Hz}, 3 \mathrm{H}), 0.90(\mathrm{~d}, J=6.6 \mathrm{~Hz}, 3 \mathrm{H}) ;{ }^{13} \mathrm{C} \mathrm{NMR}\left(126 \mathrm{MHz}, \mathrm{CDCl}_{3}\right) \delta 138.8,133.3,132.5$, $128.2,127.5,127.3,109.9,84.8,81.0,79.0,77.5,77.2,76.4,72.9,66.3,60.9,57.3,34.8,33.1$, 30.0, 26.9, 25.3, 17.2, 17.0, 12.1; IR (thin film, $\mathrm{NaCl}$ ) 3461, 2962, 2926, 1454, 1371, 1261, 1097, 800, $696 \mathrm{~cm}^{-1}$; LRMS (EI, Na) calcd for $\mathrm{C}_{26} \mathrm{H}_{42} \mathrm{O}_{6} \mathrm{Na}, 473.30 \mathrm{~m} / z(\mathrm{M}+\mathrm{Na})$; observed, $473.51(\mathrm{M}+\mathrm{Na})^{+} m / z$.

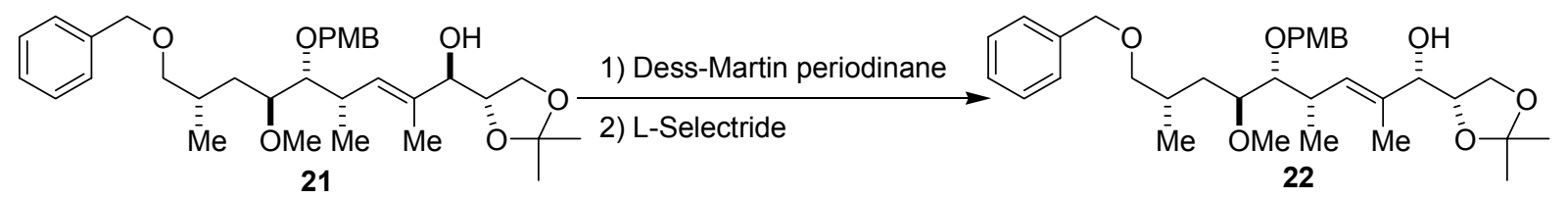

Synthesis of $E-(1 R, 4 S, 5 R, 6 S, 8 S)-5-($ paramethoxybenzyloxy)-9-(benzyloxy)-1-((S)-2,2dimethyl-1,3-dioxolan-4-yl)-6-methoxy-2,4,8-trimethylnon-2-en-1-ol, 22: To a stirring solution of a diastereomeric mixture of $\mathbf{2 1}$ and $22(32 \mathrm{mg}, 0.057 \mathrm{mmol})$ in dichloromethane (0.5 mL, reagent grade) was added 1,1,1-triacetoxy-1,1-dihydro-1,2-benziodoxol-3-(1H)-one (36 $\mathrm{mg}, 0.086 \mathrm{mmol}$ ) at room temperature. After $1 \mathrm{~h}$ of stirring, the reaction was diluted with a solution of $20 \%$ EtOAc/ hexanes $(10 \mathrm{~mL})$ and most of the solvent was removed. The residue was dissolved in $10 \mathrm{~mL}$ of EtOAc/ hexanes and washed with a 1:1 mixture of $10 \%$ $\mathrm{Na}_{2} \mathrm{~S}_{2} \mathrm{O}_{3}$ and sat. $\mathrm{NaHCO}_{3}$ until a clear organic layer was obtained. The organic layer was then washed with brine $(1 \times 10 \mathrm{~mL})$ and dried over anhydrous $\mathrm{Na}_{2} \mathrm{SO}_{4}$. The solvent was concentrated and the residue was purified by flash column chromatography eluting with $20 \%$ EtOAc/ hexanes to afford the corresponding enone which was immediately used in the 
following step. To a cooled solution of recovered enone in $600 \mu \mathrm{L}$ of $\mathrm{THF}$ at $-78{ }^{\circ} \mathrm{C}$ was added L-Selectride (1.0 M in hexanes; $173 \mu \mathrm{L}, 0.173 \mathrm{mmol})$ slowly via syringe down the side of the flask. After stirring for $30 \mathrm{~min}, 0.5 \mathrm{~mL}$ of $\mathrm{H}_{2} \mathrm{O}$ was added and the suspension was allowed to reach room temperature. All contents were loaded directly on a silica gel column, and the product was purified by flash column chromatography eluting with $20 \%$ EtOAc/ hexanes affording $28 \mathrm{mg}$ of unsaturated alcohol 22 ( $0.050 \mathrm{mmol}, \mathrm{dr} \geq 20: 1,87 \%$ over 2 steps).

\section{Data $E-(1 R, 4 S, 5 R, 6 S, 8 S)-5-($ paramethoxybenzyloxy)-9-(benzyloxy)-1-((S)-2,2-dimethyl-}

1,3-dioxolan-4-yl)-6-methoxy-2,4,8-trimethylnon-2-en-1-ol, 22: $[\alpha]_{589}{ }^{20}+6.2^{\circ}(c=0.21$, $\left.\mathrm{CHCl}_{3}\right) ;{ }^{1} \mathrm{H}$ NMR $\left(400 \mathrm{MHz}, \mathrm{CDCl}_{3}\right) \delta$ 7.34-7.25 (m, 7H), 6.88-6.83 (m, 2H), $5.29(\mathrm{dd}, J=$ 9.6, $0.8 \mathrm{~Hz}, 1 \mathrm{H}), 4.63(\mathrm{~A}$ of $\mathrm{AB}, J=10.9 \mathrm{~Hz}, 1 \mathrm{H}), 4.59(\mathrm{~B}$ of $\mathrm{AB}, J=10.9 \mathrm{~Hz}, 1 \mathrm{H}), 4.50$ (app. s, 2H), $4.12(\mathrm{dd}, J=13.9,6.0 \mathrm{~Hz}, 1 \mathrm{H}), 3.93(\mathrm{dd}, J=8.1,6.3 \mathrm{~Hz}, 1 \mathrm{H}), 3.85(\mathrm{dd}, J=7.3$, $3.3 \mathrm{~Hz}, 1 \mathrm{H}), 3.80(\mathrm{~s}, 3 \mathrm{H}), 3.67(\mathrm{dd}, J=8.1,5.8 \mathrm{~Hz}, 1 \mathrm{H}), 3.42-3.35(\mathrm{~m}, 2 \mathrm{H}), 3.35(\mathrm{~s}, 3 \mathrm{H})$, 3.27-3.18 (m, 2H), 2.55-2.46 (m, 1H), $2.40(\mathrm{~d}, J=3.3 \mathrm{~Hz}, 1 \mathrm{H}), 2.05-1.96(\mathrm{~m}, 1 \mathrm{H}), 1.79$ (ddd, $J=14.4,10.6,3.8 \mathrm{~Hz}, 1 \mathrm{H}), 1.63(\mathrm{~d}, J=1.3 \mathrm{~Hz}, 3 \mathrm{H}), 1.45(\mathrm{~s}, 3 \mathrm{H}), 1.36(\mathrm{~s}, 3 \mathrm{H}), 1.17(\mathrm{ddd}, J=$ 14.1, 10.1, 2.0 Hz, 1H), $1.04(\mathrm{~d}, J=6.6 \mathrm{~Hz}, 3 \mathrm{H}), 0.90(\mathrm{~d}, J=6.6 \mathrm{~Hz}, 3 \mathrm{H}) ;{ }^{13} \mathrm{C}$ NMR $(126$ $\left.\mathrm{MHz}, \mathrm{CDCl}_{3}\right) \delta 159.1,139.0,133.3,132.5,131.1,129.6,128.3,127.5,127.3,113.7,109.9$, $81.9,81.7,78.9,77.4,77.2,76.5,73.8,72.9,66.3,57.4,55.3,34.8,33.4,30.2,26.9,25.4$ 17.7, 17.0, 12.2; IR (thin film, $\mathrm{NaCl}$ ) 3482, 2962, 2927, 2870, 1613, 1514, 1455, 1370, 1259, 1213, 1097, 1069, 802, 696, $668 \mathrm{~cm}^{-1}$; LRMS (EI, Na) calcd for $\mathrm{C}_{33} \mathrm{H}_{48} \mathrm{O}_{7} \mathrm{Na}, 579.34 \mathrm{~m} / z(\mathrm{M}$ $+\mathrm{Na})$; observed, $579.52(\mathrm{M}+\mathrm{Na})^{+} \mathrm{m} / z$. 




17
1) Lindlar, $\mathrm{H}_{2}$

2) $\mathrm{K}_{2} \mathrm{OsO}_{4} \cdot 2 \mathrm{H}_{2} \mathrm{O}$

3) $\mathrm{NaIO}_{4}$

4) $\mathrm{NaBH}_{4}$

5) PPTS, $\mathrm{MeO}_{x} \mathrm{OMe}$

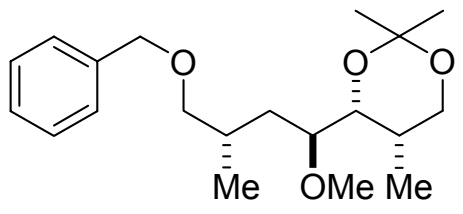

29

Structure proof:

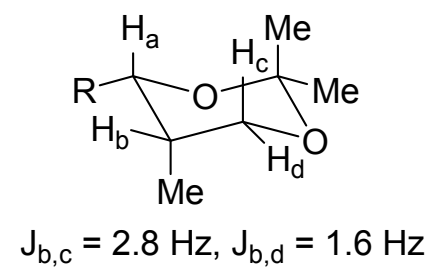

Synthesis of $(4 R, 5 S)-4-((1 S, 3 S)-4-($ benzyloxy)-1-methoxy-3-methylbutyl)-2,2,5-trimethyl-

1,3-dioxane, 29: To a flame-dried flask containing $60 \mathrm{mg}$ of alkyne $17(0.20 \mathrm{mmol})$ as a solution in $2.0 \mathrm{~mL}$ of benzene was added palladium on calcium carbonate poisoned with lead $(5 \%, 42 \mathrm{mg}, 0.020 \mathrm{mmol})$. The solution was exposed to a hydrogen atmosphere for $30 \mathrm{~min}$, and the resulting solution was filtered through celite. The solvent was removed in vacuo, and the crude product was taken on without further purification. The resulting olefin was dissolved in $653 \mu \mathrm{L}$ of acetone and $65 \mu \mathrm{L}$ of water. To it was added $7 \mathrm{mg}$ of $\mathrm{K}_{2} \mathrm{OsO}_{4} \cdot 2 \mathrm{H}_{2} \mathrm{O}$ (0.02 mmol) and $N$-morpholine- $N$-oxide $\left(60 \%\right.$ solution in $\left.\mathrm{H}_{2} \mathrm{O}, 50 \mu \mathrm{L}, 0.24 \mathrm{mmol}\right)$. The solution was left to stir overnight and sodium sulfite was subsequently added. This solution was stirred for an additional 12 hours before being filtered through a pad of silica eluting with $50 \%$ EtOAc-hexanes. The resulting diol was dissolved in $1.0 \mathrm{~mL}$ of dichloromethane and 0.5 $\mathrm{mL}$ of water. To this solution was added sodium periodate $(122 \mathrm{mg}, 0.569 \mathrm{mmol})$ and the reaction was allowed to stir $\times 12 \mathrm{~h}$ before being brought up in EtOAc $(5 \mathrm{~mL})$ and water $(5 \mathrm{~mL})$. The aqueous layer was extracted with EtOAc $(3 \times 5 \mathrm{~mL})$, and the combined organic layers were subsequently washed with brine $(1 \times 5 \mathrm{~mL})$. The solution was dried over $\mathrm{Na}_{2} \mathrm{SO}_{4}$, filtered, and all volatiles were removed in vacuo. The aldehyde was taken on without further 
purification. The resulting aldehyde was dissolved in $1.0 \mathrm{~mL}$ of $\mathrm{MeOH}$ and to it was added sodium borohydride $(22 \mathrm{mg}, 0.57 \mathrm{mmol})$. The solution was stirred $\times 2 \mathrm{~h}$ before being quenched with water. The aqueous layer was extracted with EtOAc $(3 \times 5 \mathrm{~mL})$ and the combined organics were washed with brine $(1 \times 5 \mathrm{~mL})$. The organic layer was dried over $\mathrm{Na}_{2} \mathrm{SO}_{4}$, filtered, and all volatiles were removed in vacuo. The resulting oil was filtered through a pad of silica eluting with $40 \%$ EtOAc-hexanes. The alcohol was then dissolved in $1.5 \mathrm{~mL}$ of dichloromethane and to it was added 2,2-dimethoxypropane ( $70 \mu \mathrm{L}, 0.567 \mathrm{mmol})$ and PPTS ( $3.5 \mathrm{mg}, 0.014 \mathrm{mmol})$. The solution was allowed to stir for $1 \mathrm{~h}$ before being loaded on a silica-gel column, eluting with $20 \%$ EtOAc-hexanes to afford pure acetonide 29 (42 mg, $0.13 \mathrm{mmol}, 65 \%$ yield over 5 steps) for characterization.

\section{Data for (4R,5S)-4-((1S,3S)-4-(benzyloxy)-1-methoxy-3-methylbutyl)-2,2,5-trimethyl-1,3-}

dioxane, 29: $[\alpha]_{589}{ }^{20}+3.3^{\circ}\left(c=1.68, \mathrm{CHCl}_{3}\right) ;{ }^{1} \mathrm{H} \mathrm{NMR}\left(500 \mathrm{MHz}, \mathrm{CDCl}_{3}\right) \delta$ 7.35-7.26 (m, 5H), 4.51 (app. s, 2H), 4.08 (dd, $J=11.7,2.8 \mathrm{~Hz}, 1 \mathrm{H}), 3.76$ (dd, $J=8.5,2.2 \mathrm{~Hz}, 1 \mathrm{H}), 3.60$ (dd, $J=11.7,1.6 \mathrm{~Hz}, 1 \mathrm{H}), 3.38(\mathrm{~s}, 3 \mathrm{H}), 3.34$ (dd, $J=9.1,6.0 \mathrm{~Hz}, 1 \mathrm{H}), 3.29-3.23(\mathrm{~m}, 2 \mathrm{H})$, 2.07-1.99 (m, 1H), 1.77-1.70 (m, 1H), 1.57 (ddd, $J=14.2,7.3,4.1 \mathrm{~Hz}, 1 \mathrm{H}), 1.42$ (ddd, $J=$ 14.2, 9.1, 3.5 Hz, 1H), 1.38 (s, 3H), $1.35(\mathrm{~s}, 3 \mathrm{H}), 1.12(\mathrm{~d}, J=6.9 \mathrm{~Hz}, 3 \mathrm{H}), 1.00(\mathrm{~d}, J=6.9 \mathrm{~Hz}$ $3 \mathrm{H}) ;{ }^{13} \mathrm{C} \mathrm{NMR}\left(126 \mathrm{MHz}, \mathrm{CDCl}_{3}\right) \delta 138.8,128.3,127.5,127.4,98.6,77.8,76.5,73.5,72.9$, 67.3, 57.4, 34.6, 29.9, 29.7, 29.5, 18.9, 17.5, 11.3; IR (thin film, NaCl) 2933, 2874, 1454, 1379, 1376, 1268, 1201, 1108, 1011, 930, $850 \mathrm{~cm}^{-1}$; LRMS (EI, Na) calcd for $\mathrm{C}_{20} \mathrm{H}_{32} \mathrm{O}_{4} \mathrm{Na}$, $359.23 \mathrm{~m} / \mathrm{z}(\mathrm{M}+\mathrm{Na})$; observed, $359.41(\mathrm{M}+\mathrm{Na})^{+} \mathrm{m} / \mathrm{z}$. 
<smiles>CC#C[C@H](C)[C@H](O)[C@@H](C)C[C@@H](C)COCc1ccccc1</smiles>

17

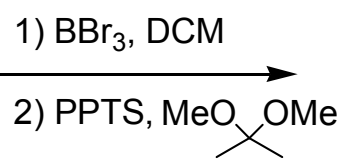

$\frac{\text { 1) } \mathrm{BBr}_{3}, \mathrm{DCM}}{\text { 2) } \mathrm{PPTS}, \mathrm{MeO} \times \mathrm{OMe}}$

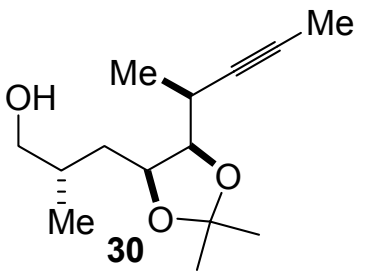

\section{Observed nOe's for structure proof of 30:}

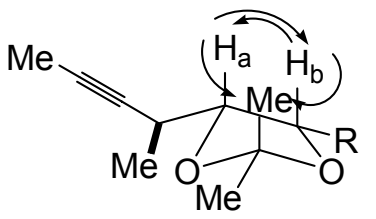

Synthesis of $(S)-3-((4 S, 5 R)-2,2-d i m e t h y l-5-((S)-p e n t-3-y n-2-y l)-1,3-d i o x o l a n-4-y l)-2-$ methylpropan-1-ol, 30: To a flame-dried flask containing alkyne 17 (100 mg, 0.329 mmol) in $1.6 \mathrm{~mL}$ of dichloromethane at $-78^{\circ} \mathrm{C}$ was added boron tribromide $(1 \mathrm{M}$ in DCM, $1.6 \mathrm{~mL}$, $1.6 \mathrm{mmol}$ ). The reaction was allowed to warm to room temperature over 1 hour before being quenched with $3 \mathrm{~mL}$ of a sat. $\mathrm{NaHCO}_{3}$ solution. The aqueous layer was extracted with EtOAc $(3 \times 5 \mathrm{~mL})$ and the combined organics were dried over sodium sulfate. The crude oil was filtered through a pad of silica eluting with $70 \%$ EtOAc-hexanes. The resulting triol was dissolved in $3.0 \mathrm{~mL}$ of dichloromethane, and to the solution was added 2,2-dimethoxypropane $(179 \mu \mathrm{L}, 1.32 \mathrm{mmol})$ and PPTS $(8 \mathrm{mg}, 0.03 \mathrm{mmol})$ at room temperature. After $30 \mathrm{~min}$ the reaction was loaded onto a silica-gel column and the product was eluted in $10 \%$ EtOAchexanes affording pure acetonide $30(15 \mathrm{mg}, 0.062 \mathrm{mmol})$ for characterization.

Data for $\quad(S)-3-((4 S, 5 R)-2,2-d i m e t h y l-5-((S)-p e n t-3-y n-2-y l)-1,3-d i o x o l a n-4-y l)-2-$ methylpropan-1-ol, 30: $[\alpha]_{589}{ }^{20}-11.25^{\circ}\left(c=0.16, \mathrm{CHCl}_{3}\right) ;{ }^{1} \mathrm{H} \mathrm{NMR}\left(500 \mathrm{MHz}, \mathrm{CDCl}_{3}\right) \delta$ 4.25 (ddd, $J 11.1,5.3,2.0 \mathrm{~Hz}, 1 \mathrm{H}), 3.92(\mathrm{dd}, J=10.1,5.3, \mathrm{~Hz}, 1 \mathrm{H}), 3.58-3.51(\mathrm{~m}, 1 \mathrm{H}), 3.49-$ $3.42(\mathrm{~m}, 1 \mathrm{H}), 2.53-2.45(\mathrm{~m}, 2 \mathrm{H}), 1.90-1.81(\mathrm{~m}, 1 \mathrm{H}), 1.76(\mathrm{~d}, J=2.5 \mathrm{~Hz}, 3 \mathrm{H}), 1.69(\mathrm{ddd}, J=$ 13.6, 6.8, 2.0 Hz, 1H), 1.57-1.50 (m, 1H), $1.44(\mathrm{~s}, 3 \mathrm{H}), 1.34(\mathrm{~s}, 3 \mathrm{H}), 1.25(\mathrm{~d}, J=6.6 \mathrm{~Hz}, 3 \mathrm{H})$, 
$0.96(\mathrm{~d}, J=6.8 \mathrm{~Hz}, 3 \mathrm{H}) ;{ }^{13} \mathrm{C} \mathrm{NMR}\left(126 \mathrm{MHz}, \mathrm{CDCl}_{3}\right) \delta 108.0,81.5,80.0,77.7,77.2,68.8$ 34.5, 34.2, 28.3, 26.3, 26.0, 19.0, 17.5, 3.4; IR (thin film, $\mathrm{NaCl}$ ) 3416, 2917, 2849, 1462, 1378, 1261, 1219, 1097, 1054, $800 \mathrm{~cm}^{-1}$; LRMS (EI, Na) calcd for $\mathrm{C}_{14} \mathrm{H}_{24} \mathrm{O}_{3} \mathrm{Na}, 263.17 \mathrm{~m} / \mathrm{z}$ $(\mathrm{M}+\mathrm{Na})$; observed, $263.20(\mathrm{M}+\mathrm{Na})^{+} \mathrm{m} / z$.

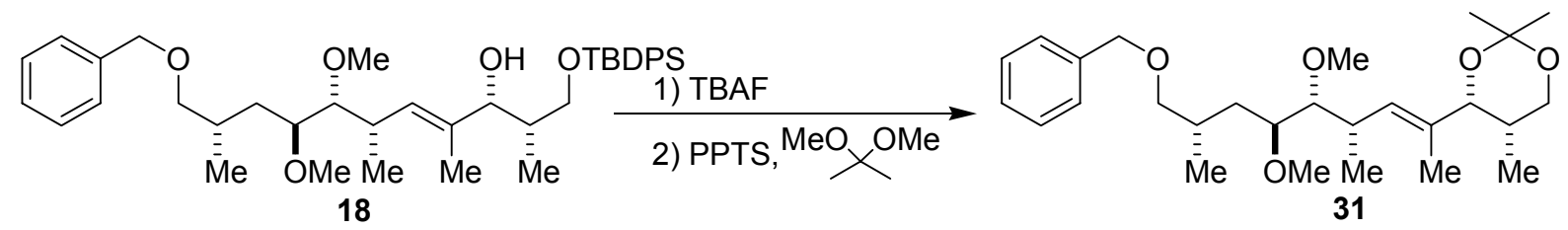

Structure proof:

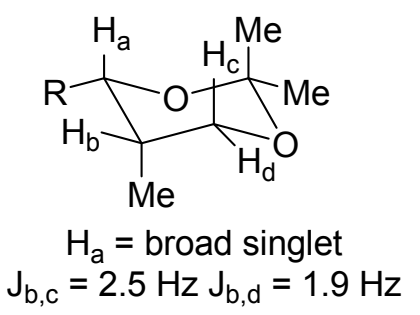

Synthesis of $(4 R, 5 S)-4-(E-(4 S, 5 R, 6 S, 8 S)-9-($ benzyloxy)-5,6-dimethoxy-4,8-dimethylnon-2en-2-yl)-2,2,5-trimethyl-1,3-dioxane, 31: Tetrabutylammonium fluoride $(273 \mu \mathrm{L}, 1 \mathrm{M}$ in THF) was added to a solution of $18(59 \mathrm{mg}, 0.091 \mathrm{mmol})$ in $0.5 \mathrm{~mL}$ of THF at room temperature. After 30 min the solution was loaded onto a pad of silica and the product was eluted with $30 \%$ EtOAc-hexanes. The resulting diol (36 mg, $0.090 \mathrm{mmol}$, 98\% yield) was taken directly onto the subsequent acetonide formation. To a solution of diol $(23 \mathrm{mg}, 0.056$ mmol) in $600 \mu \mathrm{L}$ of dichloromethane was added 2,2-dimethoxypropane (28 $\mu \mathrm{L}, 0.23 \mathrm{mmol})$ and $2 \mathrm{mg}$ of PPTS. After 30 minutes the solution was loaded onto a silica-gel column eluting with $15 \%$ EtOAc-hexanes to afford acetonide 31 ( $22 \mathrm{mg}, 0.050 \mathrm{mmol}, 89 \%$ yield).

Data for $(4 R, 5 S)-4-(E-(4 S, 5 R, 6 S, 8 S)$-9-(benzyloxy)-5,6-dimethoxy-4,8-dimethylnon-2-en2-yl)-2,2,5-trimethyl-1,3-dioxane, 31: $[\alpha]_{365}{ }^{20}+4.9^{\circ}\left(c=0.33, \mathrm{CHCl}_{3}\right) ;{ }^{1} \mathrm{H}$ NMR $(500$ 
$\left.\mathrm{MHz}, \mathrm{C}_{6} \mathrm{D}_{6}\right) \delta 7.32-7.06(\mathrm{~m}, 5 \mathrm{H}), 5.54(\mathrm{ddd}, J=10.4,1.6,1.6 \mathrm{~Hz}, 1 \mathrm{H}), 4.36(\mathrm{~A}$ of $\mathrm{AB}, J=$ $12.9 \mathrm{~Hz}, 1 \mathrm{H}), 4.35$ (B of AB, $J=12.9 \mathrm{~Hz}, 1 \mathrm{H}), 4.07$ (app. s, $1 \mathrm{H}), 3.81$ (dd, $J=11.3,2.5 \mathrm{~Hz}$, 1H), 3.47 (s, 3H), $3.46(\mathrm{dd}, J=11.6,1.9 \mathrm{~Hz}, 1 \mathrm{H}), 3.40(\mathrm{ddd}, J=10.1,2.2,2.2 \mathrm{~Hz}, 1 \mathrm{H}), 3.36$ $(\mathrm{dd}, J=9.1,5.9 \mathrm{~Hz}, 1 \mathrm{H}), 3.25-3.22(\mathrm{~m}, 1 \mathrm{H}), 3.23(\mathrm{~s}, 3 \mathrm{H}), 3.21-3.18(\mathrm{~m}, 1 \mathrm{H}), 2.69-2.61(\mathrm{~m}$, 1H), 2.28-2.20 (m, 1H), 2.04 (ddd, $J=14.2,10.0,3.8 \mathrm{~Hz}, 1 \mathrm{H}), 1.52$ (s, 3H), 1.40 (app. s, 3H), $1.33(\mathrm{ddd}, J=14.2,10.0,2.2 \mathrm{~Hz}, 1 \mathrm{H}), 1.27$ (s, 3H), 1.24-1.18 (m, 1H), 1.19 (d, $J=6.6 \mathrm{~Hz}$, $3 \mathrm{H}), 1.05(\mathrm{~d}, J=6.6 \mathrm{~Hz}, 3 \mathrm{H}), 0.99(\mathrm{~d}, J=6.9 \mathrm{~Hz}, 3 \mathrm{H}) ;{ }^{13} \mathrm{C} \mathrm{NMR}\left(126 \mathrm{MHz}, \mathrm{C}_{6} \mathrm{D}_{6}\right) \delta 139.7$, $132.1,128.5,127.7,127.4,127.3,98.7,85.5,81.7,77.0,74.0,73.0,66.4,60.4,57.0,35.1$, 33.7, 31.1, 30.7, 30.1, 19.1, 18.2, 17.1, 13.7, 11.0; IR (thin film, NaCl) 2962, 2927, 2869, 1454, 1380, 1363, 1262, 1239, 1100, 1011, $801 \mathrm{~cm}^{-1}$; LRMS (EI, Na) calcd for $\mathrm{C}_{27} \mathrm{H}_{44} \mathrm{O}_{5} \mathrm{Na}_{\text {, }}$ $471.32 \mathrm{~m} / z(\mathrm{M}+\mathrm{Na})$; observed, $471.62(\mathrm{M}+\mathrm{Na})^{+} \mathrm{m} / z$.

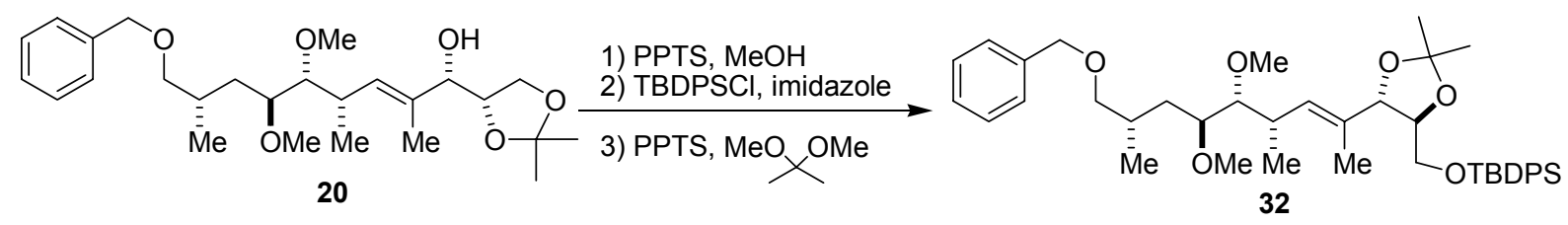

Observed nOe's for structure proof:

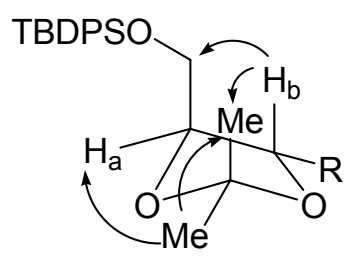

Synthesis of $(((4 S, 5 S)-5-(E-(4 S, 5 R, 6 S, 8 S)-9-(b e n z y l o x y)-5,6-d i m e t h o x y-4,8-d i m e t h y l n o n-$ 2-en-2-yl)-2,2-dimethyl-1,3-dioxolan-4-yl)methoxy)(tert-butyl)diphenylsilane, 32: To a flask containing acetonide $20(65 \mathrm{mg}, 0.14 \mathrm{mmol})$ dissolved in $2.0 \mathrm{~mL}$ of $\mathrm{MeOH}$ was added a catalytic amount of PPTS at room temperature. The resulting solution was warmed to $60{ }^{\circ} \mathrm{C}$ and stirred $\times 24$ hours. The resulting solution was cooled to room temperature before being 
filtered through a pad of silica eluting with 70\% EtOAc-hexanes. The resulting triol $(32 \mathrm{mg}$, $0.078 \mathrm{mmol}$ ) was subsequently dissolved in $0.8 \mathrm{~mL}$ of DMF. To the stirring solution was added imidazole (5.3 $\mathrm{mg}, 0.078 \mathrm{mmol})$ and TBDPSCl (20 $\mu \mathrm{L}, 0.078 \mathrm{mmol})$. The reaction was left to stir at room temperature $\times 12 \mathrm{~h}$ before being filtered through a pad of silica eluting with $40 \%$ EtOAc-hexanes. The resulting diol was subsequently dissolved in dichloromethane and to the solution was added 2,2-dimethoxypropane $(17 \mu \mathrm{L}, 0.13 \mathrm{mmol})$ and catalytic PPTS. The solution was stirred at room temperature $\times 30$ min before being loaded onto a silica-gel column eluting with 5\% EtOAc-hexanes. The resulting acetonide (19 mg, $0.027 \mathrm{mmol})$ was sufficiently pure for characterization.

\section{Data for $(((4 S, 5 S)-5-(E-(4 S, 5 R, 6 S, 8 S)-9-(b e n z y l o x y)-5,6-d i m e t h o x y-4,8-d i m e t h y l n o n-2-$} en-2-yl)-2,2-dimethyl-1,3-dioxolan-4-yl)methoxy)(tert-butyl)diphenylsilane: $[\alpha]_{589}{ }^{20}-9.6$ ${ }^{\mathrm{o}}\left(c=0.27, \mathrm{CHCl}_{3}\right) ;{ }^{1} \mathrm{H}$ NMR $\left(400 \mathrm{MHz}, \mathrm{C}_{6} \mathrm{D}_{6}\right) \delta$ 7.88-7.83 (m, 4H), 7.35-7.05 (m, $\left.11 \mathrm{H}\right)$, $5.32(\mathrm{~d}, J=10.1 \mathrm{~Hz}, 1 \mathrm{H}), 4.47$ (d, $J=8.3 \mathrm{~Hz}, 1 \mathrm{H}), 4.34$ (app. s, 2H), 3.98-3.94 (m, 1H), 3.92 (dd, $J=11.4,2.3 \mathrm{~Hz}, 1 \mathrm{H}), 3.70(\mathrm{dd}, J=11.4,4.5 \mathrm{~Hz}, 1 \mathrm{H}), 3.41$ (s, $3 \mathrm{H}), 3.30(\mathrm{~d}, J=8.8,5.6$ $\mathrm{Hz}, 1 \mathrm{H}), 3.24-3.18(\mathrm{~m}, 2 \mathrm{H}), 3.10(\mathrm{~s}, 3 \mathrm{H}), 3.04(\mathrm{dd}, J=8.6,2.5 \mathrm{~Hz}, 1 \mathrm{H}), 2.64-2.54(\mathrm{~m}, 1 \mathrm{H})$, 2.25-2.16 (m, 1H), 2.04 (ddd, $J=14.1,10.4,3.5 \mathrm{~Hz}, 1 \mathrm{H}), 1.63(\mathrm{~d}, J=1.3 \mathrm{~Hz}, 3 \mathrm{H}), 1.53(\mathrm{~s}$, $3 \mathrm{H}), 1.48(\mathrm{~s}, 3 \mathrm{H}), 1.31-1.23(\mathrm{~m}, 1 \mathrm{H}), 1.19(\mathrm{~s}, 9 \mathrm{H}), 1.13(\mathrm{~d}, J=6.6 \mathrm{~Hz}, 3 \mathrm{H}), 0.97$ (d, $J=6.8$ $\mathrm{Hz}, 3 \mathrm{H}) ;{ }^{13} \mathrm{C}$ NMR $\left(126 \mathrm{MHz}, \mathrm{C}_{6} \mathrm{D}_{6}\right) \delta$ 139.6, 136.2, 136.1, 133.9, 132.7, 132.0, 130.1, 128.5, 127.6, 127.5, 109.2, 85.0, 82.5, 81.5, 80.3, 76.9, 73.0, 63.9, 60.5, 57.2, 35.2, 33.6, 30.5, 27.6, 27.5, 27.1, 19.5, 17.3, 17.2, 12.0; IR (thin film, NaCl) 3070, 2958, 2929, 2857, 1461, 1454, $1379,1368,1260,1240,1104,1029,861,802 \mathrm{~cm}^{-1}$; LRMS (EI, Na) calcd for $\mathrm{C}_{42} \mathrm{H}_{60} \mathrm{O}_{6} \mathrm{SiNa}$, $711.42 \mathrm{~m} / z(\mathrm{M}+\mathrm{Na})$; observed, $711.68(\mathrm{M}+\mathrm{Na})^{+} \mathrm{m} / \mathrm{z}$. 


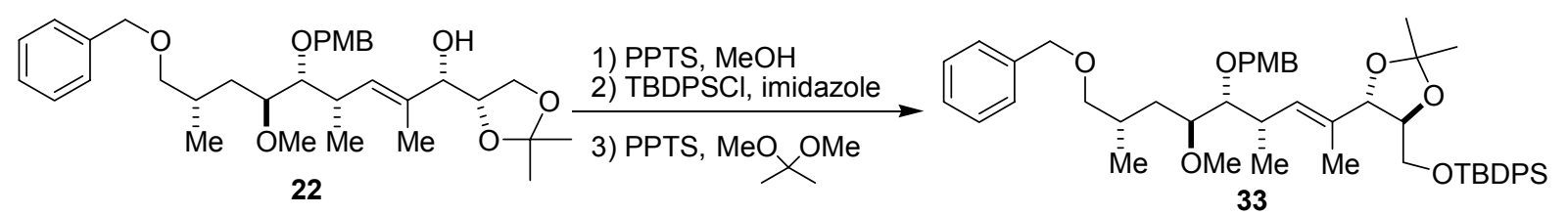

Observed nOe's for structure proof:

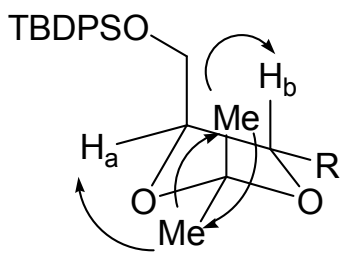

Synthesis of $\quad(((4 S, 5 S)-5-(E-(4 S, 5 R, 6 S, 8 S)-5-(4-m e t h o x y b e n z y l o x y)-9-(b e n z y l o x y)-6-$ methoxy-4,8-dimethylnon-2-en-2-yl)-2,2-dimethyl-1,3-dioxolan-4-yl)methoxy)(tert-

butyl)diphenylsilane, 33: To a flask containing acetonide 22 (43 mg, $0.077 \mathrm{mmol})$ dissolved in $2.0 \mathrm{~mL}$ of $\mathrm{MeOH}$ was added a catalytic amount of PPTS at room temperature. The resulting solution was warmed to $60{ }^{\circ} \mathrm{C}$ and stirred $\times 24$ hours. The resulting solution was cooled to room temperature before being filtered through a pad of silica eluting with $70 \%$ EtOAc-hexanes. The resulting triol $(17 \mathrm{mg}, 0.034 \mathrm{mmol})$ was subsequently dissolved in 0.4 $\mathrm{mL}$ of DMF. To the stirring solution was added imidazole (3 $\mathrm{mg}, 0.04 \mathrm{mmol})$ and TBDPSCl $(9 \mu \mathrm{L}, 0.03 \mathrm{mmol})$. The reaction was left to stir at room temperature $\times 12 \mathrm{~h}$ before being filtered through a pad of silica eluting with $40 \%$ EtOAc-hexanes. The resulting diol was subsequently dissolved in dichloromethane, and to the solution was added 2,2dimethoxypropane $(15 \mu \mathrm{L}, 0.12 \mathrm{mmol})$ and catalytic PPTS. The solution was stirred at room temperature $\times 30$ min before being loaded onto a silica-gel column eluting with $5 \%$ EtOAchexanes. The resulting acetonide 33 (17 $\mathrm{mg}, 0.025 \mathrm{mmol})$ was sufficiently pure for characterization. 
Data for (((4S,5S)-5-(E-(4S,5R,6S,8S)-5-(4-methoxybenzyloxy)-9-(benzyloxy)-6-methoxy4,8-dimethylnon-2-en-2-yl)-2,2-dimethyl-1,3-dioxolan-4-yl)methoxy)(tert-

butyl)diphenylsilane, 33: $[\alpha]_{589}{ }^{20}-6.9^{\circ}\left(c=0.29, \mathrm{CHCl}_{3}\right) ;{ }^{1} \mathrm{H} \mathrm{NMR}\left(500 \mathrm{MHz}, \mathrm{C}_{6} \mathrm{D}_{6}\right) \delta$ 7.88-7.84 (m, 4H), 7.36-7.05 (m, 13H), 6.84-6.81 (m, 2H), $5.30(\mathrm{~d}, J=9.8 \mathrm{~Hz}, 1 \mathrm{H}), 4.74(\mathrm{~A}$ of $\mathrm{AB}, J=11.0 \mathrm{~Hz}, 1 \mathrm{H}), 4.70(\mathrm{~B}$ of $\mathrm{AB}, J=11.0 \mathrm{~Hz}, 1 \mathrm{H}), 4.45(\mathrm{~d}, J=8.5, \mathrm{~Hz}, 1 \mathrm{H}), 4.34$ (app. s, 2H), 4.00-3.96 (m, 1H), 3.95-3.90 (m, 1H), $3.73(\mathrm{dd}, J=11.3,4.0 \mathrm{~Hz}, 1 \mathrm{H}), 3.37$ (d, $J$ $=8.5 \mathrm{~Hz}, 1 \mathrm{H}), 3.32-3.24(\mathrm{~m}, 2 \mathrm{H}), 3.30(\mathrm{~s}, 3 \mathrm{H}), 3.21(\mathrm{dd}, J=8.8,6.6 \mathrm{~Hz}, 1 \mathrm{H}), 3.13(\mathrm{~s}, 3 \mathrm{H})$, 2.67-2.58 (m, 1H), 2.29-2.19 (m, 1H), $2.12(\mathrm{ddd}, J=14.2,10.7,3.8 \mathrm{~Hz}, 1 \mathrm{H}), 1.64(\mathrm{~d}, J=1.3$ $\mathrm{H}, 3 \mathrm{H}), 1.52(\mathrm{~s}, 3 \mathrm{H}), 1.47(\mathrm{~s}, 3 \mathrm{H}), 1.37-1.27(\mathrm{~m}, 1 \mathrm{H}), 1.19(\mathrm{~s}, 9 \mathrm{H}), 1.16(\mathrm{~d}, J=6.6 \mathrm{~Hz}, 3 \mathrm{H})$, $0.98(\mathrm{~d}, J=6.6 \mathrm{~Hz}, 3 \mathrm{H}) ;{ }^{13} \mathrm{C} \mathrm{NMR}\left(126 \mathrm{MHz}, \mathrm{C}_{6} \mathrm{D}_{6}\right) \delta 159.7,139.6,136.2,133.9,132.5$, $132.2,132.0,130.1,129.6,128.5,127.5,113.9,109.2,82.4,82.2,82.0,80.4,76.8,74.1,73.0$ $64.0,57.3,54.8,35.4,33.7,30.5,27.6,27.5,27.1,19.5,18.0,17.3,12.1 ; \mathrm{IR}$ (thin film, $\mathrm{NaCl}$ ) 2957, 2927, 2855, 1740, 1734, 1717, 1457, 1248, 1099, $1036 \mathrm{~cm}^{-1}$; LRMS (EI, Na) calcd for $\mathrm{C}_{49} \mathrm{H}_{66} \mathrm{O}_{7} \mathrm{SiNa}, 817.46 \mathrm{~m} / z(\mathrm{M}+\mathrm{Na})$; observed, $817.65(\mathrm{M}+\mathrm{Na})^{+} \mathrm{m} / z$.

1) For the synthesis of L-(S)-glyceraldehyde acetonide, see: Hubschwerlen, C.; Specklin, J.; Higelin, J. Org. Syn. 2002, 72, 1-3. 